\title{
Exotic Minimal Surfaces
}

\author{
Francisco J. López *
}

December 29, 2018

\begin{abstract}
We prove a general fusion theorem for complete orientable minimal surfaces in $\mathbb{R}^{3}$ with finite total curvature. As a consequence, complete orientable minimal surfaces of weak finite total curvature with exotic geometry are produced. More specifically, universal surfaces (i.e., surfaces from which all minimal surfaces can be recovered) and space-filling surfaces with arbitrary genus and no symmetries.
\end{abstract}

\section{Introduction}

As usual, a surface is said to be open if it is non-compact and has empty boundary. An open Riemann surface is said to be hyperbolic if an only if it carries a negative non-constant subharmonic function. Otherwise, it is said to be parabolic. Compact Riemann surfaces with empty boundary are said to be elliptic.

Let $M$ be a Riemann surface possibly with non empty compact boundary, and let $X: M \rightarrow \mathbb{R}^{3}$ be a conformal minimal immersion. Throughout this paper we will always assume that $X$ extends as a conformal minimal immersion to an open Riemann surface containing $M$ as a proper subset. When $X$ has finite total curvature (FTC for short), Huber and Osserman theorems [5, 11] imply that $M$ has finite conformal type and the Weierstrass data of $X$ extend meromorphically to the topological ends of $M$. This simply means that $M=M^{c}-E$, where $M^{c}$ is a compact Riemann surface and $E \subset M^{c}-\partial\left(M^{c}\right)$ is a finite set, and the Weierstrass data of $X$ have no essential singularities at points of $E$. The Riemann surface $M^{c}$ is called the Osserman compactification of $M$. Likewise, a conformal complete minimal immersion $X: M \rightarrow \mathbb{R}^{3}$ is said to be of weak finite total curvature (WFTC for short) if $\left.X\right|_{\Omega}$ has finite total curvature (FTC for short) for any proper region $\Omega \subset M$ with compact boundary and finite topology.

Unlike the FTC case, there exists orientable complete minimal surfaces of WFTC with arbitrary topology and conformal type (see [8]). The aim of this paper is to present some examples of this kind of surfaces with exotic geometry.

An interesting question is whether there exists a complete minimal surface from which all minimal surfaces could be recovered. Given an open Riemann surface $N$, a complete conformal minimal immersion $Y: N \rightarrow \mathbb{R}^{3}$ is said to be universal if it passes by all compact minimal surfaces in $\mathbb{R}^{3}$. In other words, if for any compact Riemann surface $M$ with $\partial(M) \neq \emptyset$ and any conformal minimal immersion $X: M \rightarrow \mathbb{R}^{3}$, there is a sequence $\left\{M_{n}\right\}_{n \in \mathbb{N}}$ of regions in $N$ and biholomorphisms $h_{n}: M \rightarrow M_{n}, n \in \mathbb{N}$, such that $\left\{Y \circ h_{n}\right\}_{n \in \mathbb{N}} \rightarrow X$ uniformly on $M$. Our first result provides an affirmative answer to the this question (see Theorem 4.2):

${ }^{*}$ Research partially supported by MCYT-FEDER research project MTM2007-61775 and Junta de Andalucia Grant P06-FQM-01642.

2000 Mathematics Subject Classification. Primary 53A10; Secondary 49Q05, 49Q10, 53C42. Key words and phrases: Complete Minimal Surfaces, Parabolic Riemann Surfaces, Runge's Theorem, Connected Sum Constructions. 
Theorem I There exist parabolic universal minimal surfaces of WFTC.

Any universal minimal immersion $Y: N \rightarrow \mathbb{R}^{3}$ is space-filling (that is to say, $\overline{Y(N)}=\mathbb{R}^{3}$ ). As far as the author knows, all previously known space-filling complete minimal surfaces are hyperbolic, simply connected and highly symmetric. The reason why is that their construction is based either in Schwarzian reflection on a fundamental compact domain or in the classical Björling problem (see [4] for a good setting). However, in Corollary 4.1 we have shown that:

Theorem II There exists space-filling complete minimal surfaces with WFTC, arbitrary (possibly infinity) genus, parabolic conformal type and no symmetries.

Both above results are based on a general connected sum construction (or fusion theorem) for complete minimal surfaces with FTC. For a thorough exposition of the details, the following notations are required.

Given two Riemann surfaces $M$ and $M^{*}$ possibly with non empty boundary, $M^{*}$ is said to be an extension of $M$ if $M$ is a proper subset of $M^{*}, M \cap \partial\left(M^{*}\right)=\emptyset$ and $M^{*}-M^{\circ}$ contains no compact connected components that are disjoint from $\partial\left(M^{*}\right)$, where $M^{\circ}$ is the topological interior of $M$ in $M^{*}$. If $M^{*}$ is an extension of $M$ and $j: M \rightarrow M^{*}$ is the inclusion, then $j_{*}: \mathcal{H}_{1}(M, \mathbb{R}) \rightarrow \mathcal{H}_{1}\left(M^{*}, \mathbb{R}\right)$ is a group monomorphism, hence up to natural identifications $\mathcal{H}_{1}(M, \mathbb{R}) \subset \mathcal{H}_{1}\left(M^{*}, \mathbb{R}\right)$.

If $X: M \rightarrow \mathbb{R}^{3}$ is a conformal minimal immersion and $\gamma \subset M$ is an oriented closed curve, the flux of $X$ on $\gamma$ is given by $p_{X}(\gamma):=\int_{\gamma} \mu(s) d s$, where $s$ is the oriented arclength parameter on $\gamma$ and $\mu(s)$ is the conormal vector of $X$ at $\gamma(s)$ for all $s$. Recall that $\mu(s)$ is the unique unit tangent vector of $X$ at $\gamma(s)$ such that $\left\{d X\left(\gamma^{\prime}(s)\right), \mu(s)\right\}$ is a positive basis. Since $X$ is a harmonic map, $p_{X}(\gamma)$ depends only on the homology class of $\gamma$ and the well defined flux map $p_{X}: H_{1}(M, \mathbb{Z}) \rightarrow \mathbb{R}^{3}$ is a group homomorphism.

Our Fusion Theorem asserts the following (see Theorem 4.1):

Theorem III (Fusion) Let $M_{1}, M_{2}, \ldots$ be a finite or infinite sequence of pairwise disjoint Riemann surfaces with finite conformal type and non empty boundary. For each $n \in \mathbb{N}$ let $X_{n}: M_{n} \rightarrow \mathbb{R}^{3}$ be a conformal complete minimal surface of FTC.

Then, for any $\epsilon>0$ there exist an open parabolic extension $M^{*}$ of $\cup_{n} M_{n}$ and a conformal complete minimal immersion $Y: M^{*} \rightarrow \mathbb{R}^{3}$ of WFTC such that $\left\|X_{n}-\left.Y\right|_{M_{n}}\right\|_{0} \leq$ $\epsilon / n$ and $\left.p_{Y}\right|_{\mathcal{H}_{1}\left(M_{n}, \mathbb{Z}\right)}=p_{X_{n}}$, where $\|\cdot\|_{0}$ is the norm of the supremum on $M_{n}, n \in \mathbb{N}$.

The main tool for proving this theorem has been the algebraic bridge principle given in [8]. This bridge principle allows good control over the conformal structure, asymptotic behavior and flux map of the resulting surface, and supplies a natural connected sum construction for complete minimal surfaces of finite total curvature in $\mathbb{R}^{3}$. Interesting results of this kind can be found in Kapouleas [7] and Yang [12] works. Theorem III is the core of our existence result for space-filling minimal surfaces. The existence of universal minimal surfaces follows from the separability of the moduli space of complete minimal surfaces with FTC and the Fusion Theorem as well.

\section{Preliminaries on Riemann surfaces}

As usual, we denote by $\mathbb{C}, \overline{\mathbb{C}}=\mathbb{C} \cup\{\infty\}$ and $\mathbb{D}$ the complex plane, the extended complex plane and the conformal unit disc.

Given a Riemann surface $M, \partial(M)$ will denote the one dimensional topological manifold determined by the boundary points of $M$. Given $S \subset M$, write $S^{\circ}$ and $\bar{S}$ for the topological interior and the topological closure of $S$ in $M$, respectively. A proper connected subset $S \subset M$ is said to be a region if it is a topological surface with the induced topology. Open connected subsets of $M-\partial(M)$ are said to be domains of $M$. 
Given a point $P \in M-\partial(M)$, we denote by $\mu_{P}$ the harmonic measure of $M$ with respect to $P$. For any Borel measurable set $I \subset \partial(M), \mu_{P}(I)=u_{I}(P)$, where $u_{I}$ is the unique harmonic function on $M$ vanishing on the ideal boundary of $M$ and satisfying $\left.u_{I}\right|_{I}=1,\left.u_{I}\right|_{\partial(M)-I}=0$.

Definition 2.1 A Riemann surface $M$ with $\partial(M) \neq \emptyset$ is said to be parabolic if there exists $P \in$ $M-\partial(M)$ such that $\mu_{P}$ is full, i.e. $\mu_{P}(\partial M)=1$. If $N$ is an open Riemann surface, $N$ is parabolic in the classical sense if and only if $N-D^{\circ}$ is parabolic as a Riemann surface with boundary for some (hence for any) closed disc $D \subset N$.

The fact that $\mu_{P}$ is full does not depend on the interior point $P$; this follows from the maximum principle. If $P \in \Omega-\partial(\Omega)$ where $\Omega \subset M$ is a proper region, we denote by $\mu_{P}^{\Omega}$ the harmonic measure relative to $\Omega$ with respect to $P$. Notice that parabolic surfaces are exactly those on which the maximum principle holds. See [1] for a good setting.

Let $M$ and $M^{*}$ be two Riemann surfaces possibly with non empty boundary. The surface $M^{*}$ is said to be an annular extension of $M$ if $M^{*}$ is an extension of $M$ and the connected components of $M^{*}-M^{\circ}$ are either simply or doubly connected, that is to say, homeomorphic to either $[0,1] \times \mathbb{S}^{1}$ or $\overline{\mathbb{D}}-\{E\}$ for some $E \in \overline{\mathbb{D}}$. In this case $j_{*}: \mathcal{H}_{1}(M, \mathbb{R}) \rightarrow \mathcal{H}_{1}\left(M^{*}, \mathbb{R}\right)$ is an isomorphism.

\subsection{Approximation results on Riemann surfaces}

Let $N$ be a Riemann surface with $\partial(N)=\emptyset$, and let $S \subset N$ denote a subset different from $N$ and satisfying $\overline{S^{\circ}} \cap S=S$ (for instance, a finite collection of pairwise disjoint regions in $N$ ). A connected component $V$ of $N-S$ is said to be bounded if $\bar{V}$ is compact.

Definition 2.2 We denote by $\mathfrak{F}^{N}(S)$ the space of functions $f: S \rightarrow \overline{\mathbb{C}}$ being meromorphic on some open neighborhood of $S$ in $N$. If $S$ is open then $\mathfrak{F}^{N}(S)$ coincides with the space of meromorphic functions on $S$, hence does not depend on $N$ and is simply written $\mathfrak{F}(S)$. We write $\mathfrak{F}_{0}^{N}(S)$ (and $\mathfrak{F}_{0}(S)$ when $S$ is open) for the corresponding space of holomorphic functions.

All these spaces will be endowed with the $\omega(S)$-topology, namely, the topology of the uniform convergence on $S$. To be more precise, we shall say that a function $f \in \mathfrak{F}^{N}(S)$ can be uniformly approximated on $S$ by functions in $\mathfrak{F}(N)$ if there exists $\left\{f_{n}\right\}_{n \in \mathbb{N}} \subset \mathfrak{F}(N)$ such that $\left\{\left|f_{n}\right|_{S}-\right.$ $f \mid\}_{n \in \mathbb{N}} \rightarrow 0$ uniformly on $S$. In particular all $f_{n}$ have the same set $\mathcal{P}_{f}$ of poles on $S$. Likewise we define the uniform approximation of an $f \in \mathfrak{F}_{0}^{N}(S)$ by functions in $\mathfrak{F}_{0}(N)$.

A complex 1-form $\theta$ on $S$ is said to be of type $(1,0)$ if for any conformal chart $(U, z)$ on $N$ we have that $\left.\theta\right|_{U \cap S}=f(z) d z$ for some $f: U \cap S \rightarrow \overline{\mathbb{C}}$. For instance, holomorphic and meromorphic 1-forms on $N$ are of type $(1,0)$.

Definition 2.3 We denote by $\mathfrak{W}^{N}(S)$ the space of 1-foms of type $(1,0)$ on $S$ being meromorphic on some open neighborhood of $S$ in $N$. If $S$ is open then $\mathfrak{W}^{N}(S)$ coincides with the space of meromorphic 1-forms on $S$, hence does not depend on $N$ and is simply written $\mathfrak{W}(S)$. We write $\mathfrak{W}_{0}^{N}(S)$ (and $\mathfrak{W}_{0}(S)$ when $S$ is open) for the corresponding space of holomorphic 1-forms.

A 1-form $\theta \in \mathfrak{W}^{N}(S)$ can be uniformly approximated on $S$ by 1-forms in $\mathfrak{W}(N)$ if there exists $\left\{\theta_{n}\right\}_{n \in \mathbb{N}} \subset \mathfrak{W}(N)$ such that $\left\{\left.\frac{\theta_{n}-\theta}{d z}\right|_{S \cap K}\right\}_{n \in \mathbb{N}} \rightarrow 0$ in the $\omega(S \cap K)$-topology for any closed conformal disc $(K, z)$ on $N$ (we are assuming that $z: K \rightarrow z(K) \subset \mathbb{C}$ extends as a conformal parameter beyond $K$ in $N$ ). In particular all $\theta_{n}$ have the same set of poles $\mathcal{P}_{\theta}$ on $S$. As above, we say that $\left\{\left.\theta_{n}\right|_{S}\right\}_{n \in \mathbb{N}} \rightarrow \theta$ in the $\omega(S)$-topology. Likewise we define the uniform approximation of a $\theta \in \mathfrak{W}_{0}^{N}(S)$ by 1 -forms in $\mathfrak{W}_{0}(N)$.

Let $\mathfrak{D i v}(S)$ denote the free commutative group of divisors of $S$ with multiplicative notation. If $D=\prod_{i=1}^{n} Q_{i}^{n_{i}} \in \mathfrak{D i v}(S)$, where $n_{i} \in \mathbb{Z}-\{0\}$ for all $i$, the set $\left\{Q_{1}, \ldots, Q_{n}\right\}$ is said to be 
the support of $D$. Let $\mathfrak{D e g}: \mathfrak{D i v}(S) \rightarrow \mathbb{Z}$ be the group homomorphism given by the degree map $\mathfrak{D e g}\left(\prod_{j=1}^{t} Q_{j}^{n_{j}}\right)=\sum_{j=1}^{t} n_{j}$. A divisor $D \in \mathfrak{D i v}(S)$ is said to be integral if $D=\prod_{i=1}^{n} Q_{i}^{n_{i}}$ and $n_{i} \geq 0$ for all $i$. Given $D_{1}, D_{2} \in \mathfrak{D i v}(S), D_{1} \geq D_{2}$ if and only if $D_{1} D_{2}^{-1}$ is integral. For any $f \in \mathfrak{F}^{N}(S)$ we denote by $(f)_{0}$ and $(f)_{\infty}$ its associated integral divisors of zeroes and poles in $S$, respectively, and label $(f)=(f)_{0} /(f)_{\infty}$ as the divisor associated to $f$ on $S$. Likewise we define $(\theta)_{0}$ and $(\theta)_{\infty}$, and write $(\theta)=(\theta)_{0} /(\theta)_{\infty}$ for the divisor of $\theta$ in $S, \theta \in \mathfrak{W}^{N}(S)$.

We need the following extension of Runge's theorem (see [2], [9] and [10] for a good setting):

Theorem 2.1 Let $N$ be a Riemann surface with $\partial(N)=\emptyset$, and let $S \subset N$ be a finite collection of pairwise disjoint compact regions in $N$. Let $E \subset N-S$ be a subset meeting each bounded component of $N-S$ in a unique point.

Then any function $f \in \mathfrak{F}^{N}(S)$ can be uniformly approximated on $S$ by functions $\left\{f_{n}\right\}_{n \in \mathbb{N}}$ in $\mathfrak{F}(N) \cap \mathfrak{F}_{0}\left(N-\left(E \cup \mathcal{P}_{f}\right)\right)$, where $\mathcal{P}_{f}=f^{-1}(\infty) \subset S$.

\subsection{Compact Riemann surfaces}

The background of the following results can be found, for instance, in [3].

In the sequel, $R$ will denote an elliptic Riemann surface of genus $\nu \geq 1$.

Label $H_{1}(R, \mathbb{Z})$ as the $1^{s t}$ homology group with integer coefficients of $R$. Let $B=\left\{a_{j}, b_{j}\right\}_{j=1, \ldots, \nu}$ be a canonical homology basis of $H_{1}(R, \mathbb{Z})$, and write $\left\{\xi_{j}\right\}_{j=1, \ldots, \nu}$ the associated dual basis of $\mathfrak{W}_{0}(R)$, that is to say, the one satisfying that $\int_{a_{k}} \xi_{j}=\delta_{j k}, \quad j, k=1, \ldots, \nu$.

Denote by $\Pi=\left(\pi_{j k}\right)_{j, k=1, \ldots, \nu}$ the Jacobi period matrix with entries $\pi_{j k}=\int_{b_{k}} \xi_{j}, \quad j, k=$ $1, \ldots, \nu$. This matrix is symmetric and has positive definite imaginary part. We denote by $L(R)$ the lattice over $\mathbb{Z}$ generated by the $2 \nu$-columns of the $\nu \times 2 \nu$ matrix $\left(I_{\nu}, \Pi\right)$, where $I_{\nu}$ is the identity matrix of dimension $\nu$.

Finally, call $J(R)=\mathbb{C}^{\nu} / L(R)$ the Jacobian variety of $R$, which is a compact, commutative, complex, $\nu$-dimensional Lie group. Given $E \in R$, denote by $\varphi_{E}: \mathfrak{D i v}(R) \rightarrow J(R), \quad \varphi_{E}\left(\prod_{j=1}^{s} Q_{j}^{n_{j}}\right)=$ $\sum_{j=1}^{s} n_{j}{ }^{t}\left(\int_{E}^{Q_{j}} \xi_{1}, \ldots, \int_{E}^{Q_{j}} \xi_{\nu}\right)$ the Abel-Jacobi map with base point $E$, where ${ }^{t}(\cdot)$ means matrix transpose. If there is no room for ambiguity we simply write $\varphi$.

Abel's theorem asserts that $D \in \mathfrak{D i v}(R)$ is the principal divisor associated to a meromorphic function $f \in \mathfrak{W}(R)$ if and only if $\mathfrak{D e g}(D)=0$ and $\varphi(D)=0$. Jacobi's theorem says that $\varphi: R_{\nu} \rightarrow$ $J(R)$ is surjective and has maximal rank (hence a local biholomorphism) almost everywhere, where $R_{\nu}$ denotes the space of integral divisors in $\mathfrak{D i v}(R)$ of degree $\nu$.

Riemann-Roch theorem says that $r\left(D^{-1}\right)=\mathfrak{D} \mathfrak{e g}(D)-g+1+i(D)$ for any $D \in \mathfrak{D i v}(R)$, where $r\left(D^{-1}\right)$ (respectively, $i(D)$ ) is the dimension of the complex vectorial space of functions $f \in \mathfrak{F}(R)$ (respectively, 1-forms $\theta \in \mathfrak{W}(R)$ ) satisfying that $(f) \geq D^{-1}$ (respectively, $(\theta) \geq D$ ).

A point $Q \in R$ is said to be a Weierstrass point if there exists a non constant meromorphic function $h \in \mathfrak{F}(R)$ satisfying that $(h)_{\infty} \leq Q^{\nu}$. The number of Weierstrass points in $R$ lies in between $2 \nu-2$ and $\nu\left(\nu^{2}-1\right)$.

\subsection{Bridge constructions for Riemann surfaces}

A Riemann surface $M$ (possibly with non empty compact boundary) is of finite conformal type if and only if it has finite topology and is parabolic. The Osserman compactification $M^{c}$ of $M$ is obtained by filling out the conformal punctures corresponding to the topological ends of $M$. Moreover, if we attach conformal discs on the holes of $M^{c}$ we get an elliptic Riemann surface $R$ that we will call a conformal compactification of $M$. Notice that $M^{c}$ is unique up to biholomorphisms, whereas $R$ depends on the gluing process. With this language,

$$
M^{c}=R-\left(\cup_{j=1}^{b} U_{j}\right) \text { and } M=M^{c}-\left\{E_{1}, \ldots, E_{a}\right\},
$$


where $U_{j}, j=1, \ldots, b$ are open discs in $R$ with pairwise disjoint closures in $R$ and $\left\{E_{1}, \ldots, E_{a}\right\} \subset$ $M^{c}-\partial\left(M^{c}\right)$.

The following notion of conformal connected sum captures some natural bridge constructions for Riemann surfaces. We include the details just for completeness.

Let $M_{1}, M_{2}$ be two disjoint Riemann surfaces of finite conformal type and non empty boundary, and fix disjoint Jordan $\operatorname{arcs} \gamma_{i} \subset \partial\left(M_{i}\right), i=1,2$. Without loss of generality assume that $M_{1}^{c} \cap M_{2}^{c}=$ $\emptyset$ as well and write $M=M_{1} \cup M_{2}$ and $M^{c}=M_{1}^{c} \cup M_{2}^{c}$. Let $S$ be a closed conformal disc disjoint from $M^{c}$, and introduce a mark on $S$ consisting of two distinct Jordan arcs $\gamma_{1}^{\prime}, \gamma_{2}^{\prime} \subset \partial(S)$. By definition, $\Upsilon=\left(\left\{\gamma_{1}, \gamma_{2}\right\},\left\{S, \gamma_{1}^{\prime}, \gamma_{2}^{\prime}\right\}\right)$ is said to be a conformal bridge between $M_{1}$ and $M_{2}$.

The surfaces $M_{1}$ and $M_{2}$ can be connected via $\Upsilon$ as follows. Take a biholomorphism $w: S \rightarrow$ $[0,1] \times[-\delta, \delta] \subset \mathbb{C}$ such that $\gamma_{i}^{\prime}=w^{-1}\left(s_{i}\right)$, where $s_{i}$ is the segment $\{i-1\} \times[-\delta, \delta], i=1,2$ (the real number $\delta$ is uniquely determined by the mark $\left\{\gamma_{1}^{\prime}, \gamma_{2}^{\prime}\right\}$ on $\partial(S)$ ). Take a closed disc $V_{i} \subset M_{i}$ such that $\gamma_{i}=V_{i} \cap \partial\left(M_{i}\right), i=1,2$, and $V_{1} \cap V_{2}=\emptyset$, and consider biholomorphisms $w_{1}: V_{1} \rightarrow[-1,0] \times[-\delta, \delta]$ and $w_{2}: V_{2} \rightarrow[1,2] \times[-\delta, \delta]$ such that $w_{i}\left(\gamma_{i}\right)=s_{i}, i=1,2$. Then simply attach $S$ to $M$ by identifying the points $w^{-1}((i-1, t))$ and $w_{i}^{-1}((i-1, t))$ for any $t \in[-\delta, \delta]$, $i=1,2$.

Definition 2.4 We write $M_{1} \sharp \Upsilon M_{2}$ for the quotient surface, and call it a connected conformal sum (or simply, a conformal sum) of $M_{1}$ and $M_{2}$ via $\Upsilon$ (see Figure 1).

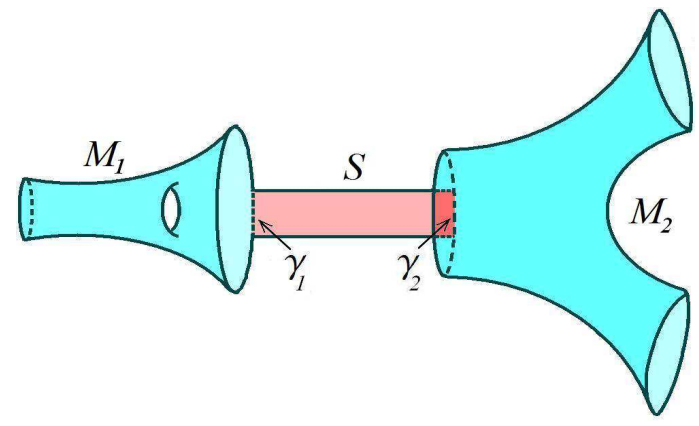

Figure 1: The surface $M_{1} \sharp \Upsilon M_{2}$

Up to the projection map to the quotient, $\gamma_{i}=\gamma_{i}^{\prime}, i=1,2$. Furthermore, $M_{1}, M_{2}$ and $S$ are subsets of $M_{1} \sharp \Upsilon M_{2}$ satisfying $M_{i} \cap S=\gamma_{i}, i=1,2$, and $M_{1} \sharp_{\Upsilon} M_{2}=M \cup S$. Adding the natural chart from $V_{1} \cup S \cup V_{2}$ onto $[-1,2] \times[-\delta, \delta]$ induced by $w, w_{1}$ and $w_{2}, M_{1} \sharp \Upsilon M_{2}$ becomes a Riemann surface of finite conformal type and non empty boundary and $H_{1}\left(M_{1} \sharp \Upsilon M_{2}, \mathbb{Z}\right)=H_{1}\left(M_{1}, \mathbb{Z}\right) \oplus \mathcal{H}_{2}\left(M_{2}, \mathbb{Z}\right)$. A conformal compactification $R_{\Upsilon}$ of $M_{1} \sharp \Upsilon M_{2}$ is said to be a conformal compactification of $M$ via $\Upsilon$. Obviously these constructions guarantee the uniqueness of neither $M_{1} \sharp_{\Upsilon} M_{2}$ nor $R_{\Upsilon}$, because they depend on the gluing processes.

This bridge construction can be used for generating parabolic Riemann surfaces of arbitrary topology. Indeed, let $\left\{M_{j}\right\}_{1 \leq j<\sigma}, \sigma \in \mathbb{N} \cup\{+\infty\}$, be sequence of pairwise disjoint Riemann surfaces of finite conformal type and non empty boundary, and call $M:=\cup_{1 \leq j<\sigma} M_{j}$. Set $W_{1}=M_{1}$, and working inductively, for each $j<\sigma$ choose a bridge $\Upsilon_{j}$ between $W_{j}$ and $M_{j+1}$ and set

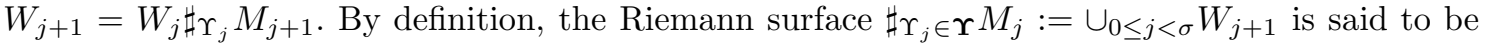

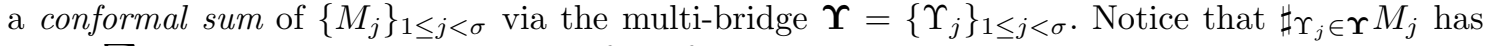
genus $\sum_{j<\sigma} \nu_{j}$, where $\nu_{j}$ is the genus of $M_{j}$ for all $j$. 
An open Riemann surface $M^{*}$ is said to be a parabolic completion of $M$ via $\Upsilon$ if $M^{*}$ is parabolic and there exists a proper topological embedding $\mathcal{I}: \sharp \Upsilon_{j} \in \Upsilon{ } M_{j} \rightarrow M^{*}$ such that $\left.\mathcal{I}\right|_{M}=\operatorname{Id}_{M}$ and $M^{*}$ is an annular extension of $\mathcal{I}\left(\sharp \Upsilon_{j} \in \Upsilon M_{j}\right)$. In particular $M^{*}$ has genus $\sum_{j<\sigma} \nu_{j}$ as well and $\mathcal{I}_{*}: H_{1}\left(\sharp \Upsilon_{j} \in \Upsilon M_{j}, \mathbb{Z}\right) \rightarrow H_{1}\left(M^{*}, \mathbb{Z}\right)$ is an isomorphism. Up to the group monomorphism induced by the inclusion map, $H_{1}\left(M_{j}, \mathbb{Z}\right)$ is a subset of $H_{1}\left(M^{*}, \mathbb{Z}\right), 1 \leq j<\sigma$, and therefore $H_{1}\left(M^{*}, \mathbb{Z}\right)$ is the direct $\operatorname{sum} \oplus_{1 \leq j<\sigma} H_{1}\left(M_{j}, \mathbb{Z}\right)$.

Lemma 2.1 Given $M=\cup_{1 \leq j<\sigma} M_{j}$ and $\Upsilon=\left\{\Upsilon_{j}\right\}_{1 \leq j<\sigma-1}$ as above, $M$ admits a parabolic completion $M^{*}$ via $\mathbf{\Upsilon}$.

Proof: Assume first that $\sigma<+\infty$. In this case $\sharp_{\Upsilon_{j} \in \Upsilon} \Upsilon_{j}$ is of finite conformal type. Let $R$ be the conformal compactification of $\sharp \Upsilon_{j} \in \Upsilon M_{j}$ and consider a finite subset $E \subset R$ containing all the ends of $M$ and meeting each component of $R-\sharp \Upsilon_{j} \in \Upsilon M_{j}$ in a unique point. It suffices to take $M^{*}=R-E$ and set $\mathcal{I}$ as the inclusion map.

Suppose now that $\sigma=+\infty$. Fix a closed disc $D \subset M_{1}-\partial\left(M_{1}\right)$ and a point $P \in D-\partial(D)$. As above, put $W_{1}=M_{1}$ and $W_{j+1}=W_{j} \sharp \Upsilon_{j} M_{j+1}$ for each $j \geq 1$. Let $c_{1}^{j} \subset \partial\left(W_{j}\right)$ and $c_{2}^{j} \subset \partial\left(M_{j+1}\right)$ be the two boundary components (closed Jordan curves) connected by $\Upsilon_{j}, j \geq 1$.

Let us construct a sequence $N_{1} \subset N_{2} \subset \ldots$ of Riemann surfaces and proper embeddings $\mathcal{I}_{j}: W_{j} \rightarrow N_{j}, j \geq 1$, such that:

(a) $\left.\mathcal{I}_{j}\right|_{W_{j-1}}=\mathcal{I}_{j-1},\left(\cup_{h>j} M_{j}\right) \cap N_{j}=\emptyset, \cup_{h \leq j} M_{j} \subset N_{j}$ and $\left.\mathcal{I}_{j}\right|_{\cup_{h \leq j} M_{j}}$ is the inclusion map, $j \geq 2$,

(b) $N_{j}$ is a Riemann surface of finite conformal type and $\partial\left(N_{j}\right)$ is a Jordan curve homologically equivalent in $N_{j}$ to $\mathcal{I}_{j}\left(c_{1}^{j}\right), j \geq 1$,

(c) $N_{j}$ is an annular extension of both $\mathcal{I}_{j}\left(W_{j}\right)$ and $N_{j-1} \sharp \Upsilon_{j-1}^{\prime} M_{j}$ for a suitable bridge $\Upsilon_{j-1}^{\prime}$ in $N_{j}$ connecting $\partial\left(N_{j-1}\right)$ and $c_{2}^{j-1}, j \geq 2$.

(d) $\mu_{P}^{N_{j}-D^{\circ}}(\partial(D))>\frac{j-1}{j}$, where $\mu_{P}^{N_{j}-D^{\circ}}$ is the harmonic measure of $N_{j}-D^{\circ}$ with respect to $P$, $j \geq 1$.

Let $R_{1}$ be an open parabolic annular extension of $M_{1}$, and notice that $R_{1}$ is biholomorphic to a finitely punctured compact Riemann surface. Without loss of generality suppose that $R_{1} \cap$ $\left(\cup_{h>1} M_{h}\right)=\emptyset$. Since $R_{1}$ is parabolic, we can find a proper region $N_{1} \subset R_{1}$ such that $N_{1}$ has just one hole (hence connected boundary), $M_{1} \subset N_{1}-\partial\left(N_{1}\right), \partial\left(N_{1}\right)$ is homologically equivalent $c_{1}^{1}$, and $N_{1}$ is an annular extension of $M_{1}$. Set $\mathcal{I}_{1}: M_{1} \rightarrow N_{1}$ the inclusion map and observe that $\mu_{P}^{N_{1}-D^{\circ}}(\partial(D))>0$. The above items hold for $j=1$.

Reasoning inductively, suppose that we have constructed $N_{j}$ and $\mathcal{I}_{j}, 1 \leq j \leq m-1$, satisfying the above properties. Take a bridge $\Upsilon_{m-1}^{\prime}$ between $N_{m-1}$ and $M_{m}$ connecting $\partial\left(N_{m-1}\right)$ and $c_{2}^{m-1}$. Let $R_{m}$ be an open parabolic annular extension of $N_{m-1} \sharp_{\Upsilon_{m-1}^{\prime}} M_{m}$. As above notice that $R_{m}$ is a finitely punctured compact Riemann surface, and without loss of generality suppose that $R_{m} \cap\left(\cup_{h>m} M_{h}\right)=\emptyset$. Let $\mathcal{I}_{m}: W_{m} \rightarrow R_{m}$ be any extension of $\mathcal{I}_{m-1}$ as a proper topological embedding satisfying that $\left.\mathcal{I}_{m}\right|_{M_{m}}=\operatorname{Id}_{M_{m}}$. Since $R_{m}$ is parabolic, there exists a proper region $N_{m} \subset R_{m}$ with just one hole such that $\mathcal{I}_{m}\left(W_{m}\right) \subset N_{m}-\partial\left(N_{m}\right), N_{m}$ is an annular extension of both $\mathcal{I}_{m}\left(W_{m}\right)$ and $N_{m-1} \sharp \Upsilon_{m-1}^{\prime} M_{m}, \partial\left(N_{m}\right)$ is homologically equivalent to $\mathcal{I}_{m}\left(c_{1}^{m}\right)$ and $\mu_{p}^{N_{m}-D^{\circ}}(\partial(D))>$ $1-1 / m$. Considering the natural embedding $\mathcal{I}_{m}: W_{m} \rightarrow N_{m}$, the induction is closed.

Set $M^{*}=\cup_{j \geq 1} N_{j}$ and $\mathcal{I}: \sharp_{\Upsilon_{j} \in \Upsilon} M_{j} \rightarrow M^{*},\left.\mathcal{I}\right|_{W_{j}}=\mathcal{I}_{j}$ for all $j$. It is not hard to check that

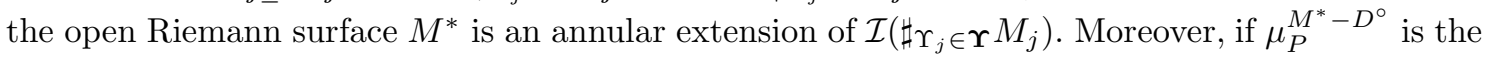
harmonic measure of $M^{*}-D^{\circ}$ with respect to $P$ then $\mu_{p}^{M^{*}-D^{\circ}}(\partial(D))=\lim _{j \rightarrow \infty} \mu_{p}^{N_{j}-D^{\circ}}(\partial(D))=$ 1 , proving that $M^{*}$ is parabolic and the lemma. 


\section{Preliminaires on minimal surfaces}

Throoughout this section, $N$ will be an open Riemann surface and $M \subset N$ a finite union of pairwise disjoint regions with compact boundary.

Definition 3.1 Let $\mathcal{E}(N)$ denote the space of conformal complete minimal immersions $X$ : $N \rightarrow \mathbb{R}^{3}$ of WFTC. Likewise, we write $\mathcal{E}_{N}(M)$ for the space of conformal complete minimal immersions $X: M \rightarrow \mathbb{R}^{3}$ of WFTC that extend as a conformal minimal immersion to some neighborhood of $M$ in $N$.

If $M$ is open then $\mathcal{E}(M)=\mathcal{E}_{M}(M)$. When $M$ has finite conformal type, $\mathcal{E}_{N}(M)$ is the space of conformal complete minimal immersions of $M$ in $\mathbb{R}^{3}$ with FTC that extend to some neighborhood of $M$ in $N$. These spaces will be endowed with the following $\mathcal{C}^{0}$ topology:

Definition 3.2 A sequence $\left\{X_{n}\right\}_{n \in \mathbb{N}} \subset \mathcal{E}_{N}(M)$ is said to converge in the $\mathcal{C}^{0}(M)$-topology to $X_{0} \in$ $\mathcal{E}_{N}(M)$ if for any proper region $\Omega \subset M$ of finite conformal type, $\left.\left\{\left.X_{n}\right|_{\Omega}\right\}_{n \in \mathbb{N}} \rightarrow X_{0}\right|_{\Omega}$ uniformly on $\Omega$. If $M$ has finite conformal type, this topology coincides with the one of uniform convergence on $M$.

Take $X \in \mathcal{E}_{N}(M)$ and write $\left(\phi_{1}, \phi_{2}, \phi_{3}\right)$ for the complex differential $\partial_{z} X$. Notice that $\partial_{z} X \in$ $\mathfrak{W}_{0}^{N}(M)^{3}$. Since $X$ is conformal and minimal, then $\phi_{1}=\frac{1}{2}(1 / g-g) \phi_{3}$ and $\phi_{2}=\frac{i}{2}(1 / g+g) \phi_{3}$, where $g \in \mathfrak{F}^{N}(M)$ and up to the stereographic projection coincides with the Gauss map of $X$. The pair $\left(g, \phi_{3}\right)$ is known as the Weierstrass representation of $X$ (see [11]).

Clearly $X(P)=X(Q)+\operatorname{Re} \int_{Q}^{P}\left(\phi_{1}, \phi_{2}, \phi_{3}\right), P, Q \in M$. The induced intrinsic metric $d s^{2}$ on $M$ and its Gauss curvature $\mathcal{K}$ are given by the expressions:

$$
d s^{2}=\sum_{j=1}^{3}\left|\phi_{j}\right|^{3}=\frac{1}{4}\left|\phi_{3}\right|^{2}\left(\frac{1}{|g|}+|g|\right)^{2}, \quad \mathcal{K}=-\left(\frac{4|d g||g|}{\left|\phi_{3}\right|\left(1+|g|^{2}\right)^{2}}\right)^{2} .
$$

The total curvature of $X$ is given by $c(X):=\int_{M} \mathcal{K} d A$, where $d A$ is the area element of $d s^{2}$, and the flux map of $X$ by the expression $p_{X}: H_{1}(M, \mathbb{Z}) \rightarrow \mathbb{R}^{3}, p_{X}(\gamma)=\operatorname{Im} \int_{\gamma} \partial_{z} X$.

By Huber, Osserman and Jorge-Meeks results $[5,11,6]$, if $X$ is complete and of FTC then $X$ is proper, $M$ has finite conformal type and the Weierstrass data of $X$ extend meromorphically to $M^{c}$.

Remark 3.1 Take $\left\{X_{n}, n \in \mathbb{N}\right\} \cup\{X\} \subset \mathcal{E}_{N}(M)$ and assume that $\left\{X_{n}\right\}_{n \in \mathbb{N}} \rightarrow X$ in $\mathcal{E}_{N}(M)$. If $\Omega \subset M$ is a proper region of finite conformal type, it is not hard to see that $\left\{\left.\left(X_{n}-X\right)\right|_{\Omega^{c}}\right\}_{n \in \mathbb{N}} \rightarrow 0$ uniformly on $\Omega^{c}$ and the Weierstrass data of $X_{n}$ converge in the $\omega\left(\Omega^{c}\right)$-topology to the ones of $X$ on $\Omega^{c}$. Indeed, just observe that by Riemann's removable singularity theorem, $X_{n}-X$ extends harmonically to the punctures of $\Omega$ for all $n$ and $\left\{X_{n}-X\right\}_{n \in \mathbb{N}} \rightarrow 0$ uniformly on $\Omega^{c}$ as well.

Let $M_{1}, M_{2}$ be two proper regions in $N$ with finite conformal type and non-empty boundary, call $M=M_{1} \cup M_{2}$ and consider an analytic regular Jordan $\operatorname{arc} \beta \subset N$ with endpoints $P_{1} \in \partial\left(M_{1}\right)$ and $P_{2} \in \partial\left(M_{2}\right)$ and otherwise disjoint from $M$ (see Figure 2). We will always assume that $\beta$ lies in an open analytic arc $\beta_{0}$ in $N$ such that $\beta_{0}-\beta \subset M-\partial(M)$. A proper region $V \subset N$ is said to be an annular extension of $M \cup \beta$ in $N$ if $M \cup \beta \subset V^{\circ}, V-\left(M^{\circ} \cup \beta\right)$ contains no connected components with compact closure that are disjoint from $\partial(V)$, and $V-\left(M^{\circ} \cup \beta\right)$ consists of a finite collection of compact annulus and once punctured closed discs. In particular, the induced homomorphism $j_{*}: H_{1}(M \cup \beta, \mathbb{Z}) \rightarrow H_{1}(V, \mathbb{Z})$ is an isomorphism, where $j: M \cup \beta \rightarrow V$ is the inclusion map. See Figure 3. A map $X: M \cup \beta \rightarrow \mathbb{R}^{3}$ is said to be smooth is $\left.X\right|_{M_{j}}, j=1,2$, and $\left.X\right|_{\beta_{0}}$ are smooth. For instance, if $Y: N \rightarrow \mathbb{R}^{3}$ is a smooth map then $X=\left.Y\right|_{M \cup \beta}$ is smooth. 


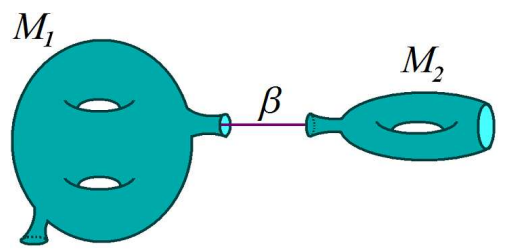

Figure 2: The surfaces $M_{1}, M_{2}$ and the curve $\beta$.
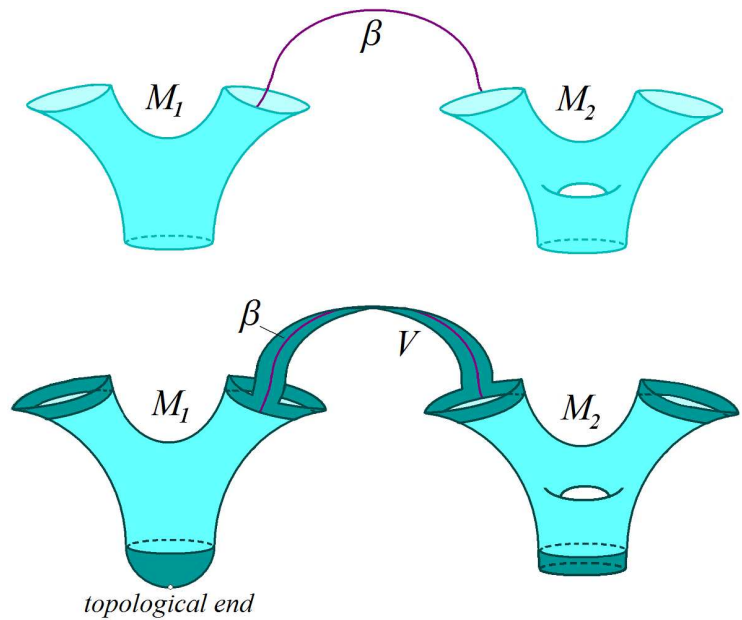

Figure 3: An annular neighborhood $V$ of $M \cup \beta$.

Definition 3.3 We denote by $\mathcal{E}_{N}(M \cup \beta)$ the space of smooth maps $X: M \cup \beta \rightarrow \mathbb{R}^{3}$ such that $X_{j}:=\left.X\right|_{M_{j}} \in \mathcal{E}_{N}\left(M_{j}\right), j=1,2$, and $\left.X\right|_{\beta}$ is a smooth immersion. This space is endowed with the $\mathcal{C}^{0}(M \cup \beta)$-topology of the uniform convergence on $M \cup \beta$.

It is clear that $\left.Y\right|_{M \cup \beta} \in \mathcal{E}_{N}(M \cup \beta)$ for all $Y \in \mathcal{E}(N)$.

Notice that $H_{1}(M \cup \beta, \mathbb{Z})=H_{1}(M, \mathbb{Z})=H_{1}\left(M_{1}, \mathbb{Z}\right) \oplus H_{1}\left(M_{2}, \mathbb{Z}\right)$, and for each $X \in \mathcal{E}(M \cup \beta)$ identify the flux map $p_{X}: H_{1}(M \cup \beta, \mathbb{Z}) \rightarrow \mathbb{R}^{3}$ of $X$ with the one of $\left.X\right|_{M}$.

The proof of the following results can be found in [8]:

Theorem 3.1 (The Algebraic Bridge Principle) Assume that $N$ is an open Riemann surface of finite conformal type and $N-(M \cup \beta)$ consists of a finite collection of pairwise disjoint once punctured conformal discs. Let $X$ be an arbitrary immersion in $\mathcal{E}_{N}(M \cup \beta)$.

Then there exists a sequence $\left\{Y_{n}\right\}_{n \in \mathbb{N}} \subset \mathcal{E}(N)$ such that $\left.p_{Y_{n}}\right|_{H_{1}(M \cup \beta)}=p_{X}$ for all $n \in \mathbb{N}$ and $\left\{\left.Y_{n}\right|_{M \cup \beta}\right\}_{n \in \mathbb{N}} \rightarrow X$ in the $\mathcal{C}^{0}(M \cup \beta)$-topology. Furthermore, if $C$ is a positive constant and $V$ an annular extension of $M \cup \beta$ in $N$, then $\left\{Y_{n}\right\}_{n \in \mathbb{N}}$ can be chosen in such a way that $d_{Y_{n}}(M \cup \beta, \partial(V)) \geq C$ for all $n$, where $d_{Y_{n}}$ is the intrinsic distance in $N$ induced by $Y_{n}$.

Theorem 3.2 (General Approximation) Let $M$ be a Riemann surface of finite conformal type and $\partial(M) \neq \emptyset$, and let $M^{*}$ be an extension of $M$ with $\partial\left(M^{*}\right)=\emptyset$. Consider an immersion $X \in \mathcal{E}_{M^{*}}(M)$ and a linear extension $q: H_{1}\left(M^{*}, \mathbb{Z}\right) \rightarrow \mathbb{R}$ of $p_{X}$.

Then, there exists a sequence of conformal complete minimal immersions $\left\{Y_{n}\right\}_{n \in \mathbb{N}} \in \mathcal{E}\left(M^{*}\right)$ such that $\left\{\left.Y_{n}\right|_{M}\right\}_{n \in \mathbb{N}} \rightarrow Y$ in the $\mathcal{C}^{0}(M)$-topology and $p_{Y_{n}}=q$. 


\section{Fusion theorems for minimal surfaces of FTC}

Parabolicity is a powerful tool because it ensures the well-posedness (existence, uniqueness, stability...) of interesting geometrical problems. Theorem 3.2 implies the existence of complete minimal surfaces of WFTC with arbitrarily prescribed (non compact) parabolic conformal structure. In particular, there exist complete parabolic minimal surfaces with arbitrary topology. Our interest resides in obtaining fusion theorems for this kind of surfaces.

Given a topological space $T$ and a continuous map $f: T \rightarrow \mathbb{R}^{3}$, we write $\|f\|_{0}=\sup \{\|f(P)\|:$ $P \in T\}$, where $\|\cdot\|$ is the Euclidean norm.

Theorem 4.1 (Fusion) Let $\left\{M_{j}\right\}_{1 \leq j<\sigma}$ be a sequence of pairwise disjoint Riemann surfaces of finite conformal type and non empty boundary, where $\sigma \in \mathbb{N} \cup\{+\infty\}$, and let $M^{*}$ be a parabolic completion of $M=\cup_{1 \leq j<\sigma} M_{j}$. Consider $X_{i} \in \mathcal{E}_{M^{*}}\left(M_{i}\right), i \geq 1$, and fix $\epsilon>0$.

Then there is $Y \in \overline{\mathcal{E}}\left(M^{*}\right)$ such that $\left\|\left.Y\right|_{M_{j}}-X_{j}\right\|_{0} \leq \epsilon / j$ and $\left.p_{Y}\right|_{H_{1}\left(M_{j}, \mathbb{Z}\right)}=p_{X_{j}}$ for all $j \geq 1$.

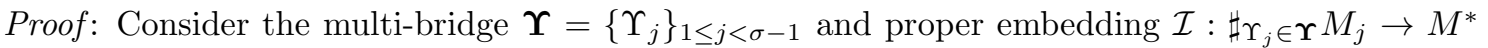
such that $\left.\mathcal{I}\right|_{M}=\operatorname{Id}_{M}$ and $M^{*}$ is an annular extension of $\mathcal{I}\left(\sharp \Upsilon_{j} \in \Upsilon M_{j}\right)$. Up to natural identifications, we will assume that $\mathcal{I}$ is the inclusion map and $M^{*}$ is an annular extension of a conformal sum $\sharp \Upsilon_{j} \in \Upsilon M_{j}$. Fix a point $P \in M_{1}$.

Like in the proof of Lemma 2.1, we can find an exhaustion $N_{1} \subset N_{2} \subset \ldots$ of $M^{*}$ by proper regions of of finite conformal type and (compact) connected boundary, and a sequence of bridges $\Upsilon_{1}^{\prime}, \Upsilon_{2}^{\prime}, \ldots$ between $N_{1}$ and $M_{2}, N_{2}$ and $M_{3}, \ldots$, such that: $N_{1}$ is an annular extension of $M_{1}$, $\cup_{h \leq j} M_{j} \subset N_{j},\left(\cup_{h>j} M_{j}\right) \cap N_{j}=\emptyset$ and $N_{j+1}$ is an annular extension of $N_{j} \sharp \Upsilon_{j}^{\prime} M_{j+1}, j \geq 1$. When $\sigma<+\infty$ the sequence ends at $N_{\sigma-1}=M^{*}$.

Let us construct $Y_{j} \in \mathcal{E}_{M^{*}}\left(N_{j}\right), 1 \leq j<\sigma$, such that: $\left\|\left.Y_{j}\right|_{M_{j}}-X_{j}\right\|_{0} \leq \frac{\epsilon}{j 2^{j}},\left.p_{Y_{j}}\right|_{H_{1}\left(M_{j}, \mathbb{Z}\right)}=p_{X_{j}}$ and $d\left(Y_{j}(P), Y_{j}\left(\partial\left(N_{j}\right)\right)\right)>j$ for all $j \geq 1$, and $\left\|\left.Y_{j}\right|_{N_{j-1}}-Y_{j-1}\right\|_{0} \leq \frac{\epsilon}{j 2^{j}}$ and $\left.p_{Y_{j}}\right|_{H_{1}\left(N_{j-1}, \mathbb{Z}\right)}=p_{Y_{j-1}}$ for all $j \geq 2$.

Indeed, by Theorem 3.2, there is $Y_{1} \in \mathcal{E}_{M^{*}}\left(N_{1}\right)$ such that $\left\|\left.Y_{1}\right|_{M_{1}}-X_{1}\right\|_{0} \leq \epsilon / 2,\left.p_{Y_{1}}\right|_{H_{1}\left(M_{1}, \mathbb{Z}\right)}=$ $p_{X_{1}}$ and $d\left(Y_{1}(P), Y_{1}\left(\partial\left(N_{1}\right)\right)\right)>1$. Reasoning inductively, suppose we have constructed $Y_{j} \in$ $\mathcal{E}_{M^{*}}\left(N_{j}\right)$ satisfying the above properties. By Theorem 3.1, there exist $Y_{j+1} \in \mathcal{E}_{M^{*}}\left(N_{j+1}\right)$ such that $\left\|\left.Y_{j+1}\right|_{M_{j+1}}-X_{j+1}\right\|_{0},\left\|\left.Y_{j+1}\right|_{N_{j}}-Y_{j}\right\|_{0} \leq \epsilon /(j+1) 2^{j+1},\left.p_{Y_{j+1}}\right|_{H_{1}\left(M_{j+1}, \mathbb{Z}\right)}=p_{X_{j+1}},\left.p_{Y_{j+1}}\right|_{H_{1}\left(N_{j}, \mathbb{Z}\right)}=$ $p_{Y_{j}}$ and $d\left(Y_{j+1}(P), Y_{j+1}\left(\partial\left(N_{j+1}\right)\right)\right)>j+1$, closing the induction.

When $\sigma<+\infty$ the immersion $Y=Y_{\sigma-1} \in \mathcal{E}\left(M^{*}\right)$ solves the theorem.

If $\sigma=+\infty$, there exists a possibly branched conformal minimal immersion $Y: M^{*} \rightarrow \mathbb{R}^{3}$ such that $\left.\left\{\left.Y_{m}\right|_{N_{j}}\right\}_{m \in \mathbb{N}} \rightarrow Y\right|_{N_{j}}$ uniformly on $N_{j}$ for any $j \geq 1$. Furthermore, $\left\|\left.Y\right|_{M_{j}}-X_{j}\right\|_{0} \leq \epsilon / j$ and $\left\|\left.Y\right|_{N_{j}}-Y_{j}\right\|_{0} \leq \epsilon /(j+1)$ for any $j \geq 1$. Let us show that $Y$ has no branch points. Indeed, let $\left(g_{j}, \phi_{3}^{j}\right)$ denote the Weierstrass data of $Y_{j}, j \geq 1$, and likewise call $\left(g, \phi_{3}\right)$ as the ones of $Y$. Obviously, $\left.\left\{g_{j}, \phi_{3}^{j}\right)\right\}_{j \in \mathbb{N}} \rightarrow\left(g, \phi_{3}\right)$ uniformly on compact subsets of $M^{*}$. Take an arbitrary $P_{0} \in M^{*}$, and consider $j_{0} \in \mathbb{N}$ such that $P_{0} \in N_{j_{0}}^{\circ}$. Up to a rigid motion, $g\left(P_{0}\right) \neq 0, \infty$, hence we can find an closed disc $D \subset N_{j_{0}}$ such that $P_{0} \in D^{\circ}$ and the functions $\left.g\right|_{D}$ and $\left.g_{j}\right|_{D}, j \in \mathbb{N}$, are holomorphic and without zeroes. Since $Y_{j}$ has no branch points, $\phi_{3}^{j}$ has no zeroes on $D$ for all $j$. By Hurwith theorem, either $\phi_{3}=0$ of $\phi_{3}$ has no zeroes on $D$ as well. In the first case the identity principle gives $\phi_{3}=0$ on $M^{*}$, contradicting that $\left\|Y-X_{1}\right\|_{0}<\epsilon$ on $M_{1}$ when $\epsilon$ is small enough, and proving that $Y_{\epsilon}$ is an immersion.

Finally, let us see that $Y$ is complete and of WFTC. By Osserman's theorem, the Gauss map of $Y_{j}$ extends meromorphically to $N_{j}^{c}, j \in \mathbb{N}$. Since $\left\|Y_{j}-\left.Y\right|_{N_{j}}\right\|_{0}$ is finite then $Y_{j}-Y$ extends harmonically to $N_{j}^{c}$ and $\left.Y\right|_{N_{j}}$ is complete and of finite total curvature for any $j$. It remains to check that $Y$ is complete. First notice that those curves in $M^{*}$ diverging to an annular end of some $N_{j}$ have infinite intrinsic length with respect to $Y$. Moreover, the fact that $d\left(Y_{j}(P), Y_{j}\left(\partial\left(N_{j}\right)\right)\right)>j$ for all $j$ implies that $\lim _{j \in \mathbb{N}} d\left(Y(P), Y\left(\partial\left(N_{j}\right)\right)\right)=+\infty$. This shows that any divergent curve in 
$M^{*}$ that does not diverge to an annular end of some $N_{j}$ have infinite intrinsic length as well, and so $Y \in \mathcal{E}\left(M^{*}\right)$. Clearly $\left.p_{Y}\right|_{H_{1}\left(M_{j}, \mathbb{Z}\right)}=p_{X_{j}}$ for all $j \geq 1$ and we are done.

This fusion theorem can be used for producing minimal surfaces with exotic geometry. We start with the following existence result for space-filling minimal surfaces:

Corollary 4.1 For each $\nu \in\{0\} \cup \mathbb{N} \cup\{\infty\}$, there exists a space-filling, open, parabolic, complete and minimal surface in $\mathbb{R}^{3}$ with genus $\nu$, WFTC and no symmetries.

Proof: Let $\left\{v_{1}, v_{2}, v_{3}\right\} \subset \mathbb{R}^{3}$ be three linearly independent unit vectors in general position, that is to say, such that $\left\langle v_{i_{1}}, v_{j_{1}}\right\rangle \neq \pm\left\langle v_{i_{2}}, v_{j_{2}}\right\rangle$ provided that $\left\{i_{1}, j_{1}\right\} \neq\left\{i_{2}, j_{2}\right\}$, where $\langle$,$\rangle is the$ Euclidean metric. Let $\left\{r_{n}: n \in \mathbb{N}\right\}$ be a bijective enumeration of $\mathbb{Q}$, and write $\Sigma_{i, n}=r_{n} v_{i}+\{u \in$ $\mathbb{R}^{3}:\left\langle u, v_{i}\right\rangle=0$ and $\left.\langle u, u\rangle \geq 1 / n^{2}\right\}, i=1,2,3, n \in \mathbb{N}$. Consider a conformal parameterization $Y_{i, n}: M_{i, n} \rightarrow \mathbb{R}^{3}$ of $\Sigma_{i, n}$, where $M_{i, n} \cap M_{j, m}=\emptyset$ provided that $(i, n) \neq(j, m)$.

Let $Z: N \rightarrow \mathbb{R}^{3}$ denote a conformal parameterization of the Chen-Gackstatter genus one minimal surface, take a closed disc $D \subset N$ and write $Z_{0}=\left.Z\right|_{N_{0}}$, where $N_{0}=N-D^{\circ}$. Recall that $N$ (hence $N_{0}$ ) has an only topological end, and $Z_{0}\left(N_{0}\right)$ is asymptotic to the classical Enneper surface. In particular, $Z_{0}\left(N_{0}\right)$ is not asymptotic to a plane. Call $N_{0, n}=N_{0} \times\{n\}$ and set $Z_{0, n}: N_{0, n} \rightarrow \mathbb{R}^{3}, Z_{0, n}((P, n))=Z_{0}(P)$ for all $n<\nu+1$. Let $\left\{Y_{j}: N_{j} \rightarrow \mathbb{R}^{3}: j \in \mathbb{N}\right\}$ denote a bijective enumeration of $\left\{Y_{i, n}: i=1,2,3, n \in \mathbb{N}\right\} \cup\left\{Z_{0, n}: n<\nu+1\right\}$.

Let $N_{j}^{*}$ be an annular neighborhood of $N_{j}$ homeomorphic to $N_{j}$, and without loss of generality suppose that $Y_{j}$ can be extended to $N_{j}^{*}, j \in \mathbb{N}$. Take a parabolic completion $M^{*}$ of $\left\{N_{j}^{*}\right\}_{j \in \mathbb{N}}$ and observe that $Y_{j} \in \mathcal{E}_{M^{*}}\left(N_{j}\right)$ for all $j$. Consider the fusion immersion $Y \in \mathcal{E}\left(M^{*}\right)$ associated to $\left\{Y_{j}\right\}_{j \in \mathbb{N}}$ and any $\epsilon>0$ via Theorem 4.1 .

It is not hard to check that $Y$ is space-filling (we leave the details to the reader). Moreover $M^{*}$ has genus $\nu$, hence it suffices to check that $Y$ has no symmetries. Reason by contradiction, and suppose there exists a rigid motion $\sigma: \mathbb{R}^{3} \rightarrow \mathbb{R}^{3}$ different from the indentity map Id and leaving invariant $Y\left(M^{*}\right)$. Call $\sigma_{0}: M^{*} \rightarrow M^{*}$ as the intrinsic isometry satisfying that $Y \circ \sigma_{0}=\sigma \circ Y$. The embedded planar annular ends of $Y$ have limit normal vector parallel to some $v_{i}, i \in\{1,2,3\}$. As $\sigma_{0}$ maps annular ends onto annular ends with the same geometry, then $\vec{\sigma}$ leaves invariant the system of vectors $\left\{ \pm v_{j}: j=1,2,3\right\}$, where $\vec{\sigma}$ is the linear isometry associated to $\sigma$. Taking into account that the vectors $v_{1}, v_{2}$ and $v_{3}$ are placed in general position, we infer that $\vec{\sigma}= \pm \mathrm{Id}$.

Assume for a moment that $\vec{\sigma}=\mathrm{Id}$, that is to say, $\sigma$ is a non trivial translation. In this case $\sigma_{0}$ takes annular planar ends on annular planar ends with the same limit normal vector. Fix $i \in\{1,2,3\}$, and for each $n \in \mathbb{N}$ let $m(n) \in \mathbb{N}$ denote the unique natural number such that $M_{i, m(n)}$ and $\sigma_{0}\left(M_{i, n}\right)$ determine the same annular end. Call $\Omega_{i, n}:=\sigma_{0}\left(M_{i, n}\right) \cap M_{i, m(n)}$ and notice that the Euclidean distance $d\left(Y\left(\Omega_{i, n}\right)-r_{m(n)} v_{i}, \Sigma_{i}\right) \rightarrow 0$ as $n \rightarrow \infty$, where $\Sigma_{i}=\left\{u:\left\langle u, v_{i}\right\rangle=0, u \neq 0\right\}$. This clearly implies that the translation vector of $\sigma$ must be orthogonal to $v_{i}$. However, this can not occur for all $i \in\{1,2,3\}$, getting a contradiction.

Suppose now that $\vec{\sigma}=-$ Id, i.e., $\sigma$ is a symmetry with respect to a point $P_{0} \in \mathbb{R}^{3}$. Reasoning as above, $\sigma$ preserves the annular ends of Enneper type. However, the Enneper type ends of $Y\left(M^{*}\right)$

lie in a neighborhood of radius $\epsilon$ of the genus one Chen-Gakstatter surface $Z(N)$, and this surface has no central symmetries. This contradiction concludes the proof.

\subsection{Universal minimal surfaces}

This section is devoted to the existence problem of universal minimal surfaces.

We start with some notations. Let $M$ be a Riemann surface of finite conformal type with $\partial(M) \neq \emptyset$, and let $N$ be an open Riemann surface. An immersion $Y \in \mathcal{E}(N)$ is said to pass by $X \in \mathcal{E}(M)$ if there exist proper regions $\left\{\Omega_{n}\right\}_{n \in \mathbb{N}}$ in $N$ and biholomorphisms $h_{n}: M \rightarrow \Omega_{n}$, 
$n \in \mathbb{N}$, such that $\left\{Y \circ h_{n}\right\}_{n \in \mathbb{N}} \rightarrow X$ in the $\mathcal{C}^{0}(M)$-topology. Note that if $Y$ passes by $X$ then $X(M) \subset \overline{Y(N)}$, but the converse is not necessarily true.

Definition 4.1 Let $N$ be an open Riemann surface $N$. An immersion $Y \in \mathcal{E}(N)$ is said to be universal if for any compact Riemann surface $M$ with non empty boundary and any conformal minimal immersion $X: M \rightarrow \mathbb{R}^{3}, Y$ passes by $X$.

The next lemma is an elementary consequence of Theorem 4.1:

Lemma 4.1 Let $\left\{Y_{i}: N_{i} \rightarrow \mathbb{R}^{3}\right\}_{1 \leq i<\sigma}$ be a sequence of conformal complete minimal immersions of $F T C$, where $\sigma \in \mathbb{N} \cup\{+\infty\}$, and assume that $\partial\left(N_{i}\right) \neq \emptyset$ for all $i$.

Then there exists an open parabolic Riemann surface $M^{*}$ and an immersion $Y \in \mathcal{E}\left(M^{*}\right)$ such that $Y$ passes by $Y_{i}$ for all $i$.

Proof: Recall that $N_{i}$ has finite conformal type for all $i$, and without loss of generality assume that $N_{i} \cap N_{j}=\emptyset, i \neq j$. Set $N_{i, j}=N_{i} \times\{j\}, h_{i, j}: N_{i} \rightarrow N_{i, j}, h_{i, j}(P)=(P, j), Y_{i, j}=Y_{i} \circ h_{i, j}^{-1}$ for all $j \in \mathbb{N}$. Write $\mathcal{Y}$ for the countable family $\left\{Y_{i, j}: 1 \leq i<\sigma, j \in \mathbb{N}\right\}$, and take a bijective enumeration $\left\{X_{j}: M_{j} \rightarrow \mathbb{R}^{3}\right\}_{j \in \mathbb{N}}$ of $\mathcal{Y}$. Label $M_{j}^{*}$ as an annular neighborhood of $M_{j}$ homeomorphic to $M_{j}^{*}$ where $X_{j}$ can be extended as a conformal minimal immersion, $j \in \mathbb{N}$, and let $M^{*}$ denote a parabolic completion of $\left\{M_{j}^{*}\right\}_{j \in \mathbb{N}}$. Note that $X_{j} \in \mathcal{E}_{M^{*}}\left(M_{j}\right)$ for all $j$, and consider the fusion immersion $Y \in \mathcal{E}\left(M^{*}\right)$ of Theorem 4.1 associated to $\left\{X_{i}\right\}_{i \in \mathbb{N}}$ and $\epsilon>0$. For any $i<\sigma$ and $j \in \mathbb{N}$, label $i_{j}$ as the unique natural such that $Y_{i, j}=X_{i_{j}}$ (hence $N_{i, j}=M_{i_{j}}$ ). As $\lim _{j \rightarrow \infty}\left\|\left.Y\right|_{M_{i_{j}}}-X_{i_{j}}\right\|_{0}=0$, then $\left\{Y \circ h_{i_{j}}\right\}_{j \in \mathbb{N}} \rightarrow Y_{i}$ in the $\mathcal{C}^{0}\left(N_{i}\right)$-topology, concluding the proof.

In order to approach the existence problem of universal minimal surfaces, we need some preliminary results on Riemann surfaces. We start with the following:

Lemma 4.2 Let $R$ be an elliptic Riemann surface, and let $V$ be an open disc in $R$. Then there is an $f_{V} \in \mathfrak{F}(R)$ all of whose branch points lie in $V$.

Proof: The proof is trivial when $R=\overline{\mathbb{C}}$. Then we will assume that $R$ has positive genus $\nu$. For the following, it is convenient to go over again the notations and results of Section 2, and specially those of Subsection 2.2.

Claim 4.1 There exists a $\tau_{0} \in \mathfrak{W}(R) \cap \mathfrak{W}_{0}^{R}(R-V)$ without zeroes in $R-V$.

Proof: Fix $E \in V$ and take a non zero $\theta \in \mathfrak{W}_{0}(R)$. Put $(\theta)=D_{1} \cdot D$, where $D_{1} \in \mathfrak{D i v}(R-V)$ and $D \in \mathfrak{D i v}(V)$. By Jacobi's theorem, we can find an open disc $U \subset V$ such that $\varphi_{E}: U_{\nu} \rightarrow \varphi_{E}\left(U_{\nu}\right)$ is a diffeomorphism, where $U_{\nu}$ is the set of divisors in $R_{\nu}$ with support in $U$. Since $J(R)$ is a compact additive Lie Group and $\varphi_{E}\left(U_{\nu}\right) \subset J(R)$ is an open subset, one has $n_{0} \varphi_{E}\left(U_{\nu}\right)=J(R)$ for large enough $n_{0} \in \mathbb{N}$ Therefore, there is $D_{2} \in U_{\nu}$ such that $\varphi_{E}\left(D_{2}^{n_{0}}\right)=\varphi_{E}\left(D_{1}\right)=\varphi_{E}\left(D_{1} E^{m}\right)$, where $m=n_{0} \nu-\mathfrak{D} \mathfrak{e} \mathfrak{g}\left(D_{1}\right)$. By Abel's theorem there exists $f_{0} \in \mathfrak{F}(R)$ such that $\left(f_{0}\right)=\frac{D_{2}^{n_{0}}}{D_{1} E^{\nu}}$. It suffices to set $\tau_{0}=f_{0} \theta$.

Fix a non Weierstrass point $Q \in V$, and label $\mathcal{U}_{Q} \subset \mathfrak{W}(R)$ as the complex vectorial subspace of meromorphic 1-forms with $(\theta) \geq Q^{-\nu-1}$. By Riemann-Roch theorem, $\operatorname{dim}_{\mathbb{C}} \mathcal{U}_{Q}=2 \nu$ and the map $\mathcal{G}: \mathcal{U}_{Q} \rightarrow \mathbb{C}^{2 \nu}, \mathcal{G}(\tau)=\left(\int_{c} \tau\right)_{c \in B}$, is a linear isomorphism.

As usual write $B=\left\{a_{j}, b_{j}\right\}_{1 \leq 1 \leq \nu}$ for a canonical basis of $H_{1}(R, \mathbb{Z})$, and choose the representative curves $a_{j}, b_{j}, j=1, \ldots, \nu$, in $R-\bar{V}$.

Claim 4.2 Let $W \subset R$ be an open disc containing $\bar{V}$ and disjoint from $a_{j}, b_{j}$ for all $j$. Then, for any function $h \in \mathfrak{F}_{0}^{R}(R-W)$ never vanishing on $R-W$, there exists $f \in \mathfrak{F}_{0}(R-\{Q\})$ never vanishing on $R-\{Q\}$ such that $\log (h / f)$ has a well defined branch on $R-W$. 
Proof: Take $\tau \in \mathcal{U}_{Q}$ such that $d h / h-\tau$ has vanishing periods along $a_{j}, b_{j}$ for all $j$, and observe that $\frac{1}{2 \pi i} \int_{a_{j}} \tau, \frac{1}{2 \pi i} \int_{b_{j}} \tau \in \mathbb{Z}$ for all $j$. Set $h_{0}=\int(d h / h-\tau) \in \mathfrak{F}_{0}^{R}(R-W)$ and $f=e^{\int \tau} \in \mathfrak{F}_{0}(R-\{Q\})$. Finally, note that $f$ never vanishes on $R-\{Q\}$ and $\log (h / f)=h_{0} \in \mathfrak{F}_{0}^{R}(R-W)$.

Let $\sigma$ be a non null exact 1-form in $\mathfrak{W}_{0}(R-\{Q\})$, and let $W \subset R$ be an open disc containing $\bar{V}$ and all the zeroes of $\sigma_{0}$ in $R-\{Q\}$.

Claim 4.3 There exists $\kappa \in \mathfrak{W}(R-\{Q\}) \cap \mathfrak{W}_{0}^{R}(R-V)$ without zeroes on $R-V$ and $g_{0} \in \mathcal{F}_{0}^{R}(R-W)$ such that $\left.\sigma\right|_{R-W}=e^{g_{0}}\left(\left.\kappa\right|_{R-W}\right)$.

Proof: Set $h=\left.\left(\sigma / \tau_{0}\right)\right|_{R-W}$, where $\tau_{0}$ is the 1-form given in Claim 4.1. If necessary, choose the representative curves $a_{j}, b_{j}, j=1, \ldots, \nu$, for $B$ in $R-W$. By the previous claim, there is $f \in \mathfrak{F}_{0}(R-\{Q\})$ never vanishing on $R-\{Q\}$ such that $g_{0}:=\log (h / f)$ is a well defined holomorphic map on $R-W$. Label $\kappa=f \tau_{0} \in \mathfrak{W}(R-\{Q\}) \cap \mathfrak{W}_{0}^{R}(R-V)$, and note that $\kappa$ has no zeroes on $R-V$. Finally, observe that $\left.\sigma\right|_{R-W}=\left.\left(e^{g_{0}} \kappa\right)\right|_{R-W} \in \mathfrak{W}_{0}^{R}(R-W)$.

Claim 4.4 The linear map $\mathcal{L}_{0}: \mathfrak{F}_{0}(R-\{Q\}) \rightarrow \mathbb{C}^{2 \nu}, \mathcal{L}_{0}(h)=\left(\int_{c} h e^{g_{0}} \kappa\right)_{c \in B}$, is surjective.

Proof: Endow $\mathfrak{F}_{0}(R-\{Q\})$ with the topology of the uniform convergence on compact subsets of $R-\{Q\}$, and observe that $\mathcal{L}_{0}$ is continuous. Take a basis $\left\{\theta_{j}\right\}_{j=1, \ldots, 2 \nu}$ of $\mathcal{U}_{Q}$, set $h_{j}=\theta_{j} /\left(\left(e^{g_{0}} \kappa\right) \in\right.$ $\mathfrak{F}_{0}^{R}(R-V)$ for each $j$, and observe that $\left\{\left(\int_{c} h_{j} e^{g_{0}} \kappa\right)_{c \in B}\right\}_{j=1, \ldots, 2 \nu}$ is a basis of $\mathbb{C}^{n}$.

On the other hand, Theorem 2.1 implies that $\mathfrak{F}_{0}(R-\{Q\})$ is dense in $\mathfrak{F}_{0}^{R}(R-W)$ with respect to the $\omega(R-W)$-topology, and so $h_{j}$ lies in the closure of $\mathfrak{F}_{0}(R-\{Q\})$ in $\mathfrak{F}_{0}^{R}(R-W)$ for all $j$. By a continuity argument $\mathcal{L}_{0}$ is surjective and we are done.

Consider $\left\{g_{n}\right\}_{n \in \mathbb{N}} \subset \mathfrak{F}_{0}(R-\{Q\})$ such that $\left.\left\{\left.g_{n}\right|_{R-W}\right\}_{n \in \mathbb{N}} \rightarrow g_{0}\right|_{R-W}$ in the $\omega(R-W)$-topology (use Theorem 2.1). Set $\mathcal{L}_{n}: \mathfrak{F}_{0}(R-\{Q\}) \rightarrow \mathbb{C}^{2 \nu}, \mathcal{L}(h)=\left(\int_{c} h e^{g_{n}} \kappa\right)_{c \in B}$, and observe that $\mathcal{L}_{n}$ is a continuous linear operator for all $n \in \mathbb{N} \cup\{0\}$. Furthermore, $\left\{\mathcal{L}_{n}\right\}_{n \in \mathbb{N}} \rightarrow \mathcal{L}_{0}$ in the weak topology, that is to say, $\left\{\mathcal{L}_{n}(h)\right\}_{n \in \mathbb{N}} \rightarrow \mathcal{L}_{0}(h)$ for all $h \in \mathfrak{F}_{0}(R-\{Q\})$. By Claim 4.4 there exists $\left\{f_{j}\right\}_{j=1, \ldots, 2 \nu} \subset \mathfrak{F}_{0}(R-\{Q\})$ such that $\left\{\mathcal{L}_{n}\left(f_{j}\right)\right\}_{j=1, \ldots, 2 \nu}$ generates $\mathbb{C}^{2 \nu}, n$ large enough (up to removing finitely many terms, for all $n \in \mathbb{N} \cup\{0\})$. Define $\mathcal{Q}_{n}: \mathbb{C}^{2 \nu} \rightarrow \mathbb{C}^{2 \nu}, \quad \mathcal{Q}_{n}\left(\left\{x_{j}\right\}_{j=1, \ldots, 2 \nu}\right)=$ $\left(\int_{c} e^{g_{n}+\sum_{j=1}^{2 \nu} x_{j} f_{j}} \kappa\right)_{c \in B}, n \in \mathbb{N} \cup\{0\}$, and notice that $\left\{\mathcal{Q}_{n}\right\}_{n \in \mathbb{N}} \rightarrow \mathcal{Q}_{0}$ as analytic maps on compact subsets of $\mathbb{C}^{2 \nu}$. Since the Jacobian of $\mathcal{Q}_{n}$ at $\mathbf{0}=(0)_{j=1, \ldots, 2 \nu}$ is different from zero for all $n \in$ $\mathbb{N} \cup\{0\}$, there exists an Euclidean ball $B_{0} \subset \mathbb{C}^{2 \nu}$ centered at $\mathbf{0}$ such that $\mathcal{Q}_{n}: B_{0} \rightarrow \mathcal{Q}_{n}\left(B_{0}\right)$ is a diffeomorphism for all $n \in \mathbb{N} \cup\{0\}$. Furthermore, as $\mathcal{Q}_{0}(\mathbf{0})=\mathbf{0}$ then $\mathbf{0} \in \mathcal{Q}_{n}\left(B_{0}\right)$ for large enough $n$ (without loss of generality, for all $n$ ). Take $\left(y_{j}^{n}\right)_{j=1, \ldots, 2 \nu} \in B_{0}$ such that $\mathcal{Q}_{n}\left(\left(y_{j}^{n}\right)_{j=1, \ldots, 2 \nu}\right)=\mathbf{0}$ and set $\sigma_{n}=e^{g_{n}+\sum_{j=1}^{2 \nu} y_{j} f_{j}} \kappa \in \mathfrak{W}_{0}(R-\{Q\}), n \in \mathbb{N}$. The 1 -form $\sigma_{n}$ have no periods and never vanish on $R-V$, hence the function $F_{n}=\int \sigma_{n} \in \mathfrak{F}_{0}^{R}(R-V)$ has no branch points on $R-V, n \in \mathbb{N}$.

To finish, fix $n_{0} \in \mathbb{N}$ and use Theorem 2.1 to find $\left\{H_{k}\right\}_{k \in \mathbb{N}} \subset \mathfrak{F}(R) \cap \mathfrak{F}_{0}(R-\{Q\})$ such that $\left\{H_{k}\right\}_{k \in \mathbb{N}} \rightarrow F_{n_{0}}$ in the $\omega(R-V)$-topology. By Hurwitz theorem, we can suppose that $d H_{n}$ never vanishes on $R-V$ for all $n$. It suffices to choose $f_{V}=H_{n}$ for some $n \in \mathbb{N}$.

Given a polynomial $\mathfrak{p}$ with complex coefficients in the variables $z$ and $w$, we denote by $\operatorname{Deg}_{z}(\mathfrak{p})$ and $\operatorname{Deg}_{w}(\mathfrak{p})$ the degree of $\mathfrak{p}$ in $z$ and $w$, respectively.

Let $R$ be an elliptic Riemann surface of genus $\nu \geq 1$. For any $f \in \mathcal{F}(R)$, write $\operatorname{Deg}(f)$ for the degree of $f$ as meromorphic function on $R$. Let $Q \in R$ be a non Weierstrass point, and for each $n \geq \nu+1$, let $f_{n} \in \mathfrak{F}(R) \cap \mathfrak{F}_{0}(R-\{Q\})$ denote a non zero function with $\operatorname{Deg}\left(f_{n}\right)=n$ and polar divisor $\left(f_{n}\right)_{\infty}=Q^{n}$. Label $z=f_{\nu+1}$ and $w=f_{\nu+2}$. We know there is an irreducible complex polynomial $\mathfrak{p}$ in the variables $z$ and $w$ with $\operatorname{Deg}_{z}(\mathfrak{p})-1=\operatorname{Deg}_{w}(\mathfrak{p})=\nu+1$ satisfying $\mathfrak{p}(z(P), w(P))=0$ for all $P \in R$. Furthermore, $R$ is biholomorphic to the algebraic curve $C_{\mathfrak{p}}:=$ $\left\{(z, w) \in \overline{\mathbb{C}}^{2}: \mathfrak{p}(z, w)=0\right\}$ (up to this biholomorphism we will consider $R=C_{\mathfrak{p}}$ ), and the pair 
$\{z, w\}$ generates the field of meromorphic functions $\mathcal{F}(R)$. The last means that any $f \in \mathfrak{F}(R)$ is of the form $f=\mathfrak{p}_{1}(z, w) / \mathfrak{p}_{2}(z, w)$ for suitable polynomials $\mathfrak{p}_{1}$ and $\mathfrak{p}_{2}$ without common factors and having $\operatorname{Deg}_{w}\left(\mathfrak{p}_{i}\right) \leq \nu$.

Remark 4.1 If $R=\overline{\mathbb{C}}$, we also have that $R \cong C_{\mathfrak{p}_{0}}:=\left\{(z, w) \in \overline{\mathbb{C}}^{2}: \mathfrak{p}_{0}(z, w)=0\right\}$ for $\mathfrak{p}_{0}(z, w)=$ $w^{2}-\left(z-a_{1}\right)\left(z-a_{2}\right)$, where $a_{1}, a_{2} \in \mathbb{C}, a_{1} \neq a_{2}$.

Definition 4.2 For each $v=(\nu, k, s) \in(\mathbb{N} \cup\{0\}) \times \mathbb{N}^{2}$, write $\mathcal{W}_{v}$ for the space of couples $(\mathfrak{p}, F)$ such that:

- $\mathfrak{p}(z, w)$ is an irreducible complex polynomial in $(z, w)$ with $\operatorname{Deg}_{z}(\mathfrak{p})-1=\operatorname{Deg}_{w}(\mathfrak{p})=\nu+1$.

- The algebraic curve $C_{\mathfrak{p}}$ has genus $\nu,(\infty, \infty)$ is the only pole of $z$ and $w$ as meromorphic functions on $C_{\mathfrak{p}}$, and $(0,0) \in C_{\mathfrak{p}}$.

- $F=\left(\left(\mathfrak{p}_{1, j}, \mathfrak{p}_{2, j}\right)\right)_{j=1,2,3}$, where $\mathfrak{p}_{1, j}$ and $\mathfrak{p}_{2, j}$ are complex polynomials in $(z, w)$ with no common factors so that $\operatorname{Deg}_{w}\left(\mathfrak{p}_{i, j}\right) \leq \nu, i=1,2, j=1,2,3$.

- Setting $f_{j}: C_{\mathfrak{p}} \rightarrow \overline{\mathbb{C}}, f_{j}(P):=\mathfrak{p}_{1, j}(z(P), w(P)) / \mathfrak{p}_{2, j}(z(P), w(P))$, we have that $\sum_{j=1}^{3} f_{j}^{2}=0$ on $C_{\mathfrak{p}}$ and $\operatorname{Deg}(g)=k$, where $g:=f_{3} /\left(f_{1}-i f_{2}\right)$.

- The polar set $E_{\mathfrak{p}, F}$ of the vectorial 1-form $F d z$ on $C_{\mathfrak{p}}$ has $s$ points, $(0,0) \notin E_{\mathfrak{p}, F}$, and $\sum_{j=1}^{3}\left|f_{j}\right|^{2}|d z|^{2}$ has no zeroes on $C_{\mathfrak{p}}-E_{\mathfrak{p}, F}$.

- The meromorphic 1-form $f_{j} d z \in \mathfrak{W}(R)$ has no real periods on $C_{\mathfrak{p}}-E_{\mathfrak{p}, F}, j=1,2,3$.

We also set $\mathcal{A}_{v}=\mathbb{R}^{3} \times \mathcal{W}_{v}$.

For any two complex polynomials $\mathfrak{p}_{1}(z, w)=\sum_{i, j} a_{i, j} z^{i} w^{j}$ and $\mathfrak{p}_{2}(z, w)=\sum_{i, j} b_{i, j} z^{i} w^{j}$, we set $d\left(\mathfrak{p}_{1}, \mathfrak{p}_{2}\right)=\sum_{i, j}\left|a_{i, j}-b_{i, j}\right|$. We endow $\mathcal{W}_{v}$ with the topology induced by the metric $d^{7} \equiv d \times(d \times d)^{3}$, and likewise equip $\mathcal{A}_{v}=\mathbb{R}^{3} \times \mathcal{W}_{v}$ with the topology induced by the metric $d_{0} \times d^{7}$, where $d_{0}$ is the Euclidean metric in $\mathbb{R}^{3}$.

Given $v=(\nu, k, s)$ and $(\mathfrak{p}, F) \in \mathcal{W}_{v}$ as above, elementary algebraic arguments show that $\operatorname{Deg}_{z}\left(\mathfrak{p}_{h, j}\right), h=1,2, j=1,2,3$, admit an universal upper bound depending only on $k$ and $\nu$. Notice also that for any $y=(x,(\mathfrak{p}, F)) \in \mathcal{A}_{v}$, the well defined immersion

$$
X_{y}: C_{p}-E_{\mathfrak{p}, F} \rightarrow \mathbb{R}^{3}, \quad X_{y}(q)=x+\operatorname{Re}\left(\int_{(0,0)}^{q} F d z\right)
$$

lies in $\mathcal{E}\left(C_{\mathfrak{p}}-E_{\mathfrak{p}, F}\right)$ for all $(\mathfrak{p}, F) \in \mathcal{W}_{v}$.

For each $v=(\nu, k, s) \in(\mathbb{N} \cup\{0\}) \times \mathbb{N}^{2}$, let $\mathcal{E}_{v}$ denote the moduli space of conformal complete minimal immersions $X: M \rightarrow \mathbb{R}^{3}$ such that $M$ is a $s$-punctured genus $\nu$ elliptic Riemann surface and $X$ has total curvature $-4 \pi k$. It is clear that $X_{y} \in \mathcal{E}_{v}$ for any $y \in \mathcal{A}_{v}$.

Equip $\mathcal{E}_{v}$ with the following topology: a sequence $\left\{X_{n}: M_{n} \rightarrow \mathbb{R}^{3}\right\}_{n \in \mathbb{N}} \subset \mathcal{E}_{v}$ is said to be convergent in the $\mathcal{C}_{*}^{0}$ topology to $X_{0}: M_{0} \rightarrow \mathbb{R}^{3}$, where $X_{0} \in \mathcal{E}_{v}$, if for any compact region $\Omega_{0} \subset M_{0}$ there exist compact regions $\Omega_{n} \subset M_{n}$ and biholomorphisms $h_{n}: \Omega_{0} \rightarrow \Omega_{n}, n \in \mathbb{N}$, such that $\left.\left\{X_{n} \circ h_{n}\right\}_{n \in \mathbb{N}} \rightarrow X_{0}\right|_{\Omega_{0}}$ in the $\mathcal{C}^{0}\left(\Omega_{0}\right)$-topology.

Lemma 4.3 The map $\Delta_{v}: \mathcal{A}_{v} \rightarrow \mathcal{E}_{v}, \Delta_{v}(y):=X_{y}$ is surjective and continuous. 
Proof: For the surjectivity, take an arbitrary immersion $X: M \rightarrow \mathbb{R}^{3}$ in $\mathcal{E}_{v}$. By Osserman's theorem, $M=R-\left\{Q_{1}, \ldots, Q_{s}\right\}$, where $R$ is an elliptic genus $\nu$ Riemann surface, and the Weierstrass data of $X$ extend meromorphically to $R$. Fix a non Weierstrass point $Q \in M$ and as above take $z, w \in \mathfrak{F}(R)$ with $\operatorname{Deg}(z)=\operatorname{Deg}(w)-1=\nu+1$ and $(z)_{\infty}=Q^{\nu+1},(w)_{\infty}=Q^{\nu+2}$. Fix also $Q_{0} \in M-\{Q\}$, and without loss of generality suppose $z\left(Q_{0}\right)=w\left(Q_{0}\right)=0$. Label $\mathfrak{p}(z, w)$ as the irreducible polynomial in $(z, w)$ such that $R=C_{\mathfrak{p}}$, and write $\partial_{z} X / d z=\left(f_{j}(z, w)\right)_{j=1,2,3}$, where $f_{j} \in \mathfrak{F}(R)$ is a rational function of the form $\mathfrak{p}_{1, j}(z, w) / \mathfrak{p}_{2, j}(z, w)$ and $\operatorname{Deg}_{w}\left(\mathfrak{p}_{i, j}\right) \leq \nu$, $i=1,2, j=1,2,3$. As the meromorphic Gauss map $g=f_{3} /\left(f_{1}-i f_{2}\right)$ has degree $k$ on $R$, then $\left(\mathfrak{p}, F=\left(\mathfrak{p}_{1, j}, \mathfrak{p}_{2, j}\right)_{j=1,2,3}\right) \in \mathcal{W}_{v}$. It is clear that $X=\Delta_{v}\left(\left(X\left(Q_{0}\right),(\mathfrak{p}, F)\right)\right)$.

To check that $\Delta_{v}$ is continuous, take $\left\{y_{n}=\left(x_{n},\left(\mathfrak{p}_{n}, F_{n}\right)\right)\right\}_{n \in \mathbb{N} \cup\{0\}} \in \mathcal{A}_{v}$ such that $\left\{y_{n}\right\}_{n \in \mathbb{N}} \rightarrow$ $y_{0}$, and fix an arbitrary compact region $\Omega_{0} \subset C_{\mathfrak{p}_{0}}-E_{\mathfrak{p}_{0}, F_{0}}$. We have to find compact regions $\Omega_{n} \subset C_{\mathfrak{p}_{n}}-E_{\mathfrak{p}_{n}, F_{n}}$ and biholomorphisms $h_{n}: \Omega_{0} \rightarrow \Omega_{n}$, such that $\left.\left\{X_{y_{n}} \circ h_{n}\right\}_{n \in \mathbb{N}} \rightarrow X_{y_{0}}\right|_{\Omega_{0}}$ in the $\mathcal{C}^{0}\left(\Omega_{0}\right)$-topology.

Remark 4.2 Recall that $z$ and $w$ are meromorphic functions on $C_{\mathfrak{p}_{n}}=\left\{(z, w) \in \bar{c}^{2}: \mathfrak{p}_{n}(z, w)=0\right\}$ for all $n \in \mathbb{N} \cup\{0\}$, hence they depend on $n$. This lack of notation does not affect our exposition.

Let $U_{0}$ be an open subset of $C_{\mathfrak{p}_{0}}-\left(\{(0,0)\} \cup \Omega_{0}\right)$ containing $E_{\mathfrak{p}_{0}, F_{0}}$. By Lemma 4.2, there is a meromorphic function $z_{0}: C_{\mathfrak{p}_{0}} \rightarrow \overline{\mathbb{C}}$ with all its branch points in $U_{0}$. Write $z_{0}=\mathfrak{q}_{1}(z, w) / \mathfrak{q}_{2}(z, w)$, where $\mathfrak{q}_{1}, \mathfrak{q}_{2}$ are polynomials with no common factors and $\operatorname{Deg}_{w}\left(\mathfrak{q}_{i}\right) \leq \nu, i=1,2$, and choose $w_{0}$ any function in $\{z, w\}$ so that $\left\{z_{0}, w_{0}\right\}$ generates $\mathfrak{F}\left(C_{\mathfrak{p}_{0}}\right)$. Let $z^{l} w^{j}$ be the effective monomial (i.e., with non zero coefficient) in $\mathfrak{q}_{1}$ and $\mathfrak{q}_{2}$ with maximum degree as meromorphic function on $C_{\mathfrak{p}_{0}}$. Since $\operatorname{Deg}(w)-1=\operatorname{Deg}(z)=\nu+1$ and $\operatorname{Deg}_{w}\left(\mathfrak{q}_{i}\right) \leq \nu, i=1,2$, this monomial always exists and is unique. Furthermore, as $z$ and $w$ have an unique pole at the same point (namely, $(\infty, \infty))$ of $C_{\mathfrak{p}_{0}}$, then $\operatorname{Deg}\left(z_{0}\right)=l(\nu+1)+j(\nu+2)$. The same argument shows that $z_{n}: C_{\mathfrak{p}_{n}} \rightarrow \overline{\mathbb{C}}$, $z_{n}=\mathfrak{q}_{1}(z, w) / \mathfrak{q}_{2}(z, w)$, has $\operatorname{Deg}\left(z_{n}\right)=\operatorname{Deg}\left(z_{0}\right)$ as meromorphic function on $C_{\mathfrak{p}_{n}}$ for $n$ large enough (without loss of generality, for all $n \in \mathbb{N}$ ).

In the sequel we write $a=\operatorname{Deg}\left(z_{n}\right)$ (which does not depend on $n$ ) and $E_{n}=E_{\mathfrak{p}_{n}, F_{n}}, n \in\{0\} \cup \mathbb{N}$. We also label $B_{n}$ as the branch point set of $z_{n}$ on $C_{\mathfrak{p}_{n}}$ for all $n \in\{0\} \cup \mathbb{N}$. For any $P \in C_{\mathfrak{p}_{0}}$, denote $b_{P}$ as the branching number of $z_{0}: C_{\mathfrak{p}_{0}} \rightarrow \overline{\mathbb{C}}$ at $P$, and for each $\zeta \in \overline{\mathbb{C}}$ write $a_{\zeta}=\sum_{P \in z_{0}^{-1}(\zeta)} b_{P}$.

Choose for each $\zeta \in z_{0}\left(B_{0} \cup E_{0}\right)$ an open disc $D_{\zeta} \subset \overline{\mathbb{C}}$ centered at $\zeta$ so that:

- $\left\{\bar{D}_{\zeta}: \zeta \in z_{0}\left(B_{0} \cup E_{0}\right)\right\}$ is a family of pairwise disjoint closed discs,

- $z_{0}^{-1}\left(D_{\zeta}\right)$ consists of $a-a_{\zeta}$ conformal discs,

- if $P \in z_{0}^{-1}(\zeta)$ and $U_{P}$ is the connected component of $z_{0}^{-1}\left(D_{\zeta}\right)$ containing $P$, then $\left.z_{0}\right|_{U_{P}}$ : $U_{P} \rightarrow D_{\zeta}$ is a branched covering of $b_{P}$ sheets, and

- $\bar{U}_{P} \subset U_{0}$ when $P \in E_{0} \cup B_{0}$.

Since $\left\{d\left(\mathfrak{p}_{n}, \mathfrak{p}_{0}\right)\right\}_{n \in \mathbb{N}} \rightarrow 0, B_{0}$ is the limit set of $B_{n}$ as $n \rightarrow \infty$ in $\overline{\mathbb{C}}^{2}$. In other words, if $\left\{P_{n}\right\}_{n \in \mathbb{N}} \subset \overline{\mathbb{C}}^{2}$ is a convergent sequence such that $P_{n} \in B_{n}$ for all $n$, then its limit lies in $B_{0}$, and any point of $B_{0}$ is the limit of a sequence of this kind. Likewise, if we write $F_{n}=\left(\left(\mathfrak{p}_{1, j}^{n}, \mathfrak{p}_{2, j}^{n}\right)\right)_{j=1,2,3}$ one has $\left\{d\left(\mathfrak{p}_{i, j}^{n}, \mathfrak{p}_{i, j}^{0}\right)\right\}_{n \in \mathbb{N}} \rightarrow 0$ for all $i, j$, and so $E_{0}$ is the limit set of $\left\{E_{n}\right\}_{n \in \mathbb{N}}$ in $\overline{\mathbb{C}}^{2}$ as well.

For each $P \in z_{0}^{-1}\left(z_{0}\left(B_{0} \cup E_{0}\right)\right)$, choose $Q_{P}^{n} \in z_{n}^{-1}\left(z_{0}(P)\right), n \in \mathbb{N}$, so that $\left\{Q_{P}^{n}\right\}_{n \in \mathbb{N}} \rightarrow P$ as points of $\overline{\mathbb{C}}^{2}$. By elementary topology, and up to removing finitely many terms of the sequence $\left\{y_{n}\right\}_{n \in \mathbb{N}}$ if necessary, we can suppose that:

(i) $z_{n}^{-1}\left(D_{\zeta}\right)$ is a collection of $a-a_{\zeta}$ pairwise disjoint open discs on $C_{\mathfrak{p}_{n}}$ for all $\zeta \in z_{0}\left(B_{0} \cup E_{0}\right)$ and $n \in \mathbb{N} \cup\{0\}$. 
(ii) For any $P \in z_{0}^{-1}\left(z_{0}\left(B_{0} \cup E_{0}\right)\right)$ and $n \in \mathbb{N} \cup\{0\},\left.z_{n}\right|_{U_{P}^{n}}: U_{P}^{n} \rightarrow D_{z_{0}(P)}$ is a branched covering of $b_{P}$ sheets, where $U_{P}^{n}$ is the component of $z_{n}^{-1}\left(D_{z_{0}(P)}\right)$ containing $Q_{P}^{n}{ }^{1}$

Set $W=\overline{\mathbb{C}}-\cup_{\zeta \in z_{0}\left(B_{0} \cup E_{0}\right)} D_{\zeta}, W_{n}=z_{n}^{-1}(W) \subset C_{\mathfrak{p}_{n}}$ and $\pi_{n}:=\left.z_{n}\right|_{W_{n}}: W_{n} \rightarrow W, n \in \mathbb{N} \cup\{0\}$. Fix $\zeta_{0} \in W$ and choose $P_{0}^{n} \in z_{n}^{-1}\left(\zeta_{0}\right), n \in \mathbb{N} \cup\{0\}$, so that $\left\{P_{0}^{n}\right\}_{n \in \mathbb{N}} \rightarrow P_{0}^{0}$ as points of $\overline{\mathbb{C}}^{2}$. Basic monodromy arguments give that $\left(\pi_{n}\right)_{*}\left(\Pi_{1}\left(W_{n}\right)\right)=\left(\pi_{0}\right)_{*}\left(\Pi_{1}\left(W_{0}\right)\right) \subset \Pi_{1}(W)$, where $\Pi_{1}\left(W_{n}\right)$ is the fundamental group of $W_{n}$ with base point $P_{0}^{n}, \Pi_{1}(W)$ is the one of $W$ with base point $\zeta_{0}$, and $\left(\pi_{n}\right)_{*}: \Pi_{1}\left(W_{n}\right) \rightarrow \Pi_{1}(W)$ is the group homomorphism induced by $\pi_{n}, n \in \mathbb{N}$.

For each $n \in \mathbb{N}$, let $\lambda_{n}: W_{0} \rightarrow W_{n}$ denote the unique biholomorphism such that $\lambda_{n}\left(P_{0}^{n}\right)=P_{0}^{0}$ and $z_{n} \circ \lambda_{n}=\left.z_{0}\right|_{W_{0}}$.

Label $J=\left\{P \in z_{0}^{-1}\left(B_{0} \cup E_{0}\right): b_{P}=0\right\}$, and notice that $\left.z_{n}\right|_{U_{P}^{n}}: U_{P}^{n} \rightarrow D_{z_{0}(P)}$ is a biholomorphism for all $P \in J$ and $n \in \mathbb{N}$. Call $V_{n}=\cup_{P \in J} U_{P}^{n}$ and $\hat{W}_{n}=W_{n} \cup V_{n}, n \in \mathbb{N} \cup\{0\}$. Let $\hat{\lambda}_{n}: \hat{W}_{0} \rightarrow \hat{W}_{n}$ denote the natural extension of $\lambda_{n}$ satisfying $z_{n} \circ \hat{\lambda}_{n}=\left.z_{0}\right|_{\hat{W}_{0}}, n \in \mathbb{N}$. Since $\cup_{P \in E_{0} \cup B_{0}} \bar{U}_{P} \subset U_{0}$, then $\Omega_{0} \subset \hat{W}_{0}-\partial\left(\hat{W}_{0}\right)$. Set $\Omega_{n}=\hat{\lambda}_{n}\left(\Omega_{0}\right)$ and write $h_{n}=\left.\hat{\lambda}_{n}\right|_{\Omega_{0}}: \Omega_{0} \rightarrow \Omega_{n}$, $n \in \mathbb{N}$. The facts that $\left\{d\left(\mathfrak{p}_{n}, \mathfrak{p}_{0}\right)\right\}_{n \in \mathbb{N}} \rightarrow 0$ and $\left\{d\left(\mathfrak{p}_{i, j}^{n}, \mathfrak{p}_{i, j}^{0}\right)\right\}_{n \in \mathbb{N}} \rightarrow 0$ for all $i, j$, imply that $\left.\left\{w \circ \hat{\lambda}_{n}\right\}_{n \in \mathbb{N}} \rightarrow w\right|_{\hat{W}_{0}}$ and $\left\{F_{n}\left(z \circ \hat{\lambda}_{n}, w \circ \hat{\lambda}_{n}\right)\right\}_{n \in \mathbb{N}} \rightarrow F_{0}\left(\left.z\right|_{\hat{W}_{0}},\left.w\right|_{\hat{W}_{0}}\right)$ in the $\omega\left(\hat{W}_{0}\right)$-topology. Taking into account that $\left\{x_{n}\right\}_{n \in \mathbb{N}} \rightarrow x_{0}$, we deduce that $\left.\left\{X_{y_{n}} \circ h_{n}\right\}_{n \in \mathbb{N}} \rightarrow X_{y_{0}}\right|_{\Omega_{0}}$ in the $\mathcal{C}^{0}\left(\Omega_{0}\right)$-topology, concluding the proof.

Now we can state the main result of this subsection.

Theorem 4.2 (Existence of universal surfaces) There exist parabolic complete universal minimal surfaces of WFTC in $\mathbb{R}^{3}$.

Proof: Set $\mathcal{A}=\cup_{v \in(\mathbb{N} \cup\{0\}) \times \mathbb{N}^{2}} \mathcal{A}_{v}$ and $\mathcal{E}=\cup_{v \in(\mathbb{N} \cup\{0\}) \times \mathbb{N}^{2}} \mathcal{E}_{v}$ endowed with the corresponding direct sum topologies, and define $\Delta: \mathcal{A} \rightarrow \mathcal{E},\left.\quad \Delta\right|_{\mathcal{A}_{v}}=\Delta_{v}$.

Notice that $\mathcal{A}_{v}$ is separable, take a dense countable subset $\mathcal{D}_{v} \subset \mathcal{A}_{v}$ and denote by $\mathcal{S}_{v}=\Delta_{v}\left(\mathcal{D}_{v}\right)$.

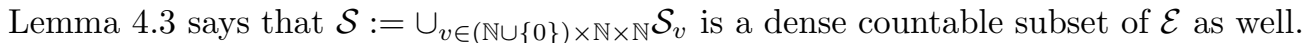

For each $X: M \rightarrow \mathbb{R}^{3}$ in $\mathcal{S}$, fix a countable basis $B_{X}$ of the topology on $M$ formed by open discs bounded by Jordan curves in $M$, and call $\mathcal{S}_{X}=\left\{\left.X\right|_{M-D}: D \in B_{X}\right\}$. Finally set $\mathcal{S}_{0}=\cup_{X \in \mathcal{S}} \mathcal{S}_{X}$.

By Lemma 4.1, there exists an open parabolic Riemann surface $M^{*}$ and an immersion $Y \in$ $\mathcal{E}\left(M^{*}\right)$ passing by $X$ for all $X \in \mathcal{S}_{0}$. Let us show that $Y$ is universal.

Let $M_{0}$ be a compact genus $\nu$ Riemann surface with non empty boundary, label $s>0$ as the number of components in $\partial\left(M_{0}\right)$. Let $X_{0}: M_{0} \rightarrow \mathbb{R}^{3}$ be a conformal minimal immersion that extends as a conformal minimal immersion to some open Riemann surface $N$ containing $M_{0}$. Let $M_{0}^{*}$ be a compact annular extension of $M_{0}$ in $N$, and construct a conformal compactification $R$ of $M_{0}^{*}$. Consider a finite subset $E \subset R-M_{0}$ so that $R-E$ is an annular extension of $M_{0}$ and notice that $X_{0} \in \mathcal{E}_{R-E}\left(M_{0}\right)$. Then take a sequence $\left\{X_{n}\right\}_{n \in \mathbb{N}} \subset \mathcal{E}(R-E)$ converging to $X_{0}$ in the $\mathcal{C}^{0}\left(M_{0}\right)$-topology (use Theorem 3.2). Note that $X_{n} \in \mathcal{E}_{v_{n}}$, where $v_{n}=\left(\nu, k_{n}, s\right)$ for some $k_{n} \in \mathbb{N}$. Fix $Q_{0} \in M_{0}$, and use Lemma 4.3 to find $y_{n}=\left(X_{n}\left(Q_{0}\right),\left(\mathfrak{p}_{n}, F_{n}\right)\right) \in \mathcal{A}_{v_{n}}$ such that $X_{n}=\Delta_{v_{n}}\left(y_{n}\right)$. By the density of $\mathcal{S}_{v_{n}}$ in $\mathcal{E}_{v_{n}}$ (see Lemma 4.3), there exists $\left\{\hat{X}_{j, n}: N_{j, n} \rightarrow \mathbb{R}^{3}\right\}_{j \in \mathbb{N}} \subset \mathcal{S}_{v_{n}}$, regions $W_{j, n} \subset N_{j, n}$ and biholomorphisms $h_{j, n}: M_{0} \rightarrow W_{j, n}, j \in \mathbb{N}$, such that $\left.\left\{\hat{X}_{j, n} \circ h_{j, n}\right\}_{j \in \mathbb{N}} \rightarrow X_{n}\right|_{M_{0}}$ in the $C^{0}\left(M_{0}\right)$-topology. Choose a disc $D_{j, n} \in B_{X_{j, n}}$ disjoint from $W_{j, n}$, call $X_{j, n}=\left.\hat{X}_{j, n}\right|_{N_{j, n}-D_{j, n}} \in$ $\mathcal{S}_{X_{j, n}} \subset \mathcal{S}_{0}$ and observe that $\left.\left\{X_{j, n} \circ h_{j, n}\right\}_{j \in \mathbb{N}} \rightarrow X_{n}\right|_{M_{0}}$ in the $C^{0}\left(M_{0}\right)$-topology too. Finally, take $j_{n} \in \mathbb{N}$ such that $\left\|X_{j_{n}, n} \circ h_{j_{n}, n}-\left.X_{n}\right|_{M_{0}}\right\|_{0}<1 / n$ and label $h_{n}=h_{j_{n}, n}, n \in \mathbb{N}$. Since $\left\{X_{j_{n}, n} \circ h_{n}\right\}_{n \in \mathbb{N}} \rightarrow X_{0}$ in the $\mathcal{C}^{0}\left(M_{0}\right)$-topology and $Y$ passes by $X_{j_{n}, n}$ for all $n$, then $Y$ passes by $X_{0}$ and we are done.

\footnotetext{
${ }^{1}$ Notice that $U_{P}=U_{P}^{0}$ for all $P \in z_{0}^{-1}\left(z_{0}\left(B_{0} \cup E_{0}\right)\right)$.
} 


\section{References}

[1] L. V. Ahlfors, L. Sario, Riemann surfaces. Princeton Univ. Press, Princeton, New Jersey, 1960.

[2] E. Bishop, Subalgebras of functions on a Riemann surface. Pacific J. Math 8 (1958), 29-50.

[3] H. M. Farkas, I. Kra, Riemann surfaces. Graduate Texts in Math., 72, Springer Verlag, Berlin, 1980.

[4] J. A. Galvez, P. Mira, Dense solutions to the Cauchy problem for minimal surfaces. Bull. Braz. Math. Soc. 35 (2004), 387-394.

[5] A. Huber, On subharmonic functions and differential geometry in the large. Comment. Math. Helv., 32, 13-72 (1957).

[6] L. P. M. Jorge, W. H. Meeks III: The topology of complete minimal surfaces of finite total Gaussian curvature. Topology, Vol. 2 (1983), 203-221.

[7] N. Kapouleas, Complete embedded minimal surfaces of finite total curvature. J. Differential Geom., 47 (1997), no. 1, 95-169.

[8] F. J. Lopez, Uniform approximation by algebraic minimal surfaces in $\mathbb{R}^{3}$. Preprint, http://arxiv.org/abs/0903.3209

[9] S. Scheinberg, Uniform approximation by functions analytic on a Riemann surface. Ann. of Math., 108 (1978), 257-298.

[10] S. Scheinberg, Uniform approximation by meromorphic functions having prescribed poles. Math. Ann., 243 (1979), 83-93.

[11] R. Osserman, A survey of minimal surfaces. Dover Publications, New York, second edition, 1986.

[12] S.-D. Yang, A connected sum construction for complete minimal surfaces of finite total curvature. Comm. Anal. Geom., 9 (2001), no. 1, 115-167.

\section{FRANCISCO J. LOPEZ}

Departamento de Geometría y Topología

Facultad de Ciencias, Universidad de Granada

18071 - GRANADA (SPAIN)

e-mail: fjlopez@ugr.es 